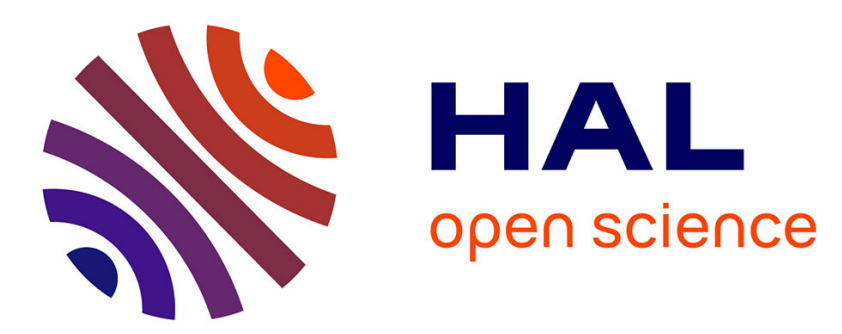

\title{
Asymptotic Models and Impedance Conditions for Highly Conductive Sheets in the Time-Harmonic Eddy Current Model \\ Victor Péron
}

\section{- To cite this version:}

Victor Péron. Asymptotic Models and Impedance Conditions for Highly Conductive Sheets in the Time-Harmonic Eddy Current Model. SIAM Journal on Applied Mathematics, 2019, 79 (6), pp. 2242-2264. 10.1137/17M1152498 . hal-01505612v2

\section{HAL Id: hal-01505612 \\ https://hal.inria.fr/hal-01505612v2}

Submitted on 4 Mar 2018

HAL is a multi-disciplinary open access archive for the deposit and dissemination of scientific research documents, whether they are published or not. The documents may come from teaching and research institutions in France or abroad, or from public or private research centers.
L'archive ouverte pluridisciplinaire HAL, est destinée au dépôt et à la diffusion de documents scientifiques de niveau recherche, publiés ou non, émanant des établissements d'enseignement et de recherche français ou étrangers, des laboratoires publics ou privés. 


\title{
ASYMPTOTIC MODELS AND IMPEDANCE CONDITIONS FOR HIGHLY CONDUCTIVE SHEETS IN THE TIME-HARMONIC EDDY CURRENT MODEL
}

\author{
VICTOR PÉRON
}

\begin{abstract}
This work is concerned with the time-harmonic eddy current problem for a medium with a highly conductive thin sheet. We present asymptotic models and impedance conditions up to the second order of approximation for the electromagnetic field. The conditions are derived asymptotically for vanishing sheet thickness $\varepsilon$ where the skin depth is scaled like $\varepsilon$. The first order condition is the perfect electric conductor boundary condition. The second order condition turns out to be a Poincaré-Steklov map between tangential components of the magnetic field and the electric field.
\end{abstract}

\section{CONTENTS}

1. Introduction 2

2. The mathematical model and asymptotic models with transmission conditions 4

2.1. Notations 4

2.2. The time-harmonic eddy current problem 4

2.3. Magnetic field formulation 6

2.4. Guideline on the derivation of equivalent models and impedance conditions 6

3. Main results. Asymptotic models up to order $2 \quad 8$

3.1. Equivalent model of order 1 for the magnetic field 8

3.2. Equivalent model of order 2 for the magnetic field 9

3.3. Equivalent models and impedance transmission conditions for the electric field 10

3.4. Regularized variational formulation for the electric field in the second order model 11

3.5. Proof of Lemma 3.6 13

4. Variational formulations for the electric field. Stability and convergence results $\quad 14$

$\begin{array}{ll}\text { 4.1. Strong form of equations } & 14\end{array}$

4.2. Variational framework $\quad 15$

$\begin{array}{ll}\text { 4.3. Variational problems } & 15\end{array}$

5. A multiscale expansion for the magnetic field 18

5.1. Expansion of differential operators inside the conductor 19

5.2. Equations for the coefficients of the magnetic field 20

$\begin{array}{ll}\text { 5.3. First terms of the asymptotics } & 20\end{array}$

5.4. Further notes for deriving the second order condition 22

References $\quad 24$

Date: October 4, 2017.

1991 Mathematics Subject Classification. 35C20, 35J25, 41A60.

Key words and phrases. Eddy Current Problem, Impedance Transmission Conditions, Thin Conducting Sheets, Asymptotic Expansions. 


\section{INTRODUCTION}

Many electronic devices feature thin plates or sheets of metal supplying efficient electromagnetic shielding. Due to their high conductivity and large aspect ratio of the plates the shielding properties are reached with a minimum use of metal. Precisely their large aspect ratio makes the numerical simulation of such devices more of a challenge, especially if standard methods like the finite element method (FEM) shall be applied. If the sheet is thin and, if, moreover, the magnetic fields decay rapidly inside the layer due to high conductivities (the skin effect), then meshes with very small cells are required. The numerical modeling is much simplified if the thin sheet is replaced by transmission conditions on its mid-surface. Meshes with much larger cells can be used with the so called impedance transmission conditions, which relate the electric and magnetic fields on both sides of the interface.

The eddy current problem has become an important research topic worldwide since several decades $[21,20,5,18,22,6,14,1,9,30]$. In this paper we address the issue of impedance transmission conditions (ITCs) for highly conductive and thin sheets for the eddy current problem in three dimensions. The concept of ITCs is rather classical in the context of electromagnetic wave propagation phenomena. Already in 1902 Levi-Civita introduced impedance transmission conditions [19] (see also [3, 33]) for Maxwell's equations. He postulated that the electric field is continuous over the interface whereas the magnetic field has a discontinuity, which is proportional to the sheet thickness and conductivity. As variation of the conditions by Levi-Civita the so called shielding element [25] has been introduced. In an alternative way the so called thin layer boundary condition $[34,18,22,15,12]$ are derived taking into account the boundary layer behaviour of the solution. In this way, the thin layer boundary conditions exhibit jumps in both the electric and magnetic field and involve sheet thickness and conductivity as arguments of hyperbolic functions.

ITCs fit naturally with finite element methods [28, 29, 4, 16] as well as boundary integral formulations [17, 24, 32], where for the latter only the interface has to be discretized. ITCs with drastically reduced error levels can systematically be derived by an asymptotic analysis of the Maxwell's equations with thin conducting sheets where the sheet thickness $\varepsilon$ tends to zero. For example, for the eddy current model in 2D the family ITC-2-N [31], in which the conductivity is scaled like $1 / \varepsilon^{2}$ and $N$ corresponds to the order, where the convergence of the modeling error outside the sheet is like $\varepsilon^{N+1}$ in their respective asymptotic regime. This convergence is not always robust in terms of the conductivity [30]. However, it turns out that the ITC-2-0 and ITC-2-1 conditions are robust and these conditions can be used from very low to very high frequencies.

Since most electromagnetic devices require the modeling in three dimensions we aim to derive rigorously reduced models for the time-harmonic eddy current problem in three dimensions. We consider the asymptotic regime in which the conductivity is scaled like $1 / \varepsilon^{2}$ in order to obtain robust conditions as the ITC-2-1 conditions in 2D. We consider the general case of curved thin sheets where the magnetic permeability may take different values inside and outside the sheet. In this paper we derive asymptotic models and ITCs up to the second order of approximation for the eddy current problem. We prove well-posedness, stability and convergence results for these models. A main contribution of this study is a stability result for the second order asymptotic model. The proof of this result is based on a regularized variational formulation together with a Helmholtz decomposition and a regularity result, Section 3. 
This work is concerned essentially with theoretical objectives. The numerical pertinence of ITCs have already been shown for the Maxwell equations in [27]. In [27], second order ITCs have been proposed for the time-harmonic Maxwell equations in three dimensions for vanishing sheet thickness $\varepsilon$ where the skin depth is kept proportional to $\varepsilon$. These conditions appear as generalized Poincaré-Steklov maps between tangential components of the magnetic field and the electric field, and they are of Wentzell type involving second order surface differential operators. Here, we use techniques to derive impedance conditions for electromagnetic models in 3D including thin layers, see e.g. [10, 27].

There are several differences between this work and the work in Ref. [27] since all the asymptotic models are different. It is worth noting that the second order ITCs in [27] do not apply to the eddy current problem since these conditions are available only for both strictly positive permittivities and conductivities whereas one considers in this work zero conductivities outside the sheet and zero permittivities. In this work we do not adopt the same approach as in [27] (which is based on an electric field formulation and an asymptotic expansion for the electric field) in order to derive ITCs, since one can not obtain all the new asymptotic models for the eddy current problem in this way. Contrary to [27], we proceed in this work with a magnetic field formulation and a multi-scale expansion for the magnetic field. First, we derive explicitly the first terms of this expansion from which we obtain asymptotic models and impedance conditions up to the second order of approximation for the magnetic field in the eddy current problem. The limit models for the magnetic field appear as tangential vector potentials. The second order ITCs for the magnetic field turns out to be surface divergence maps between tangential components and normal components of the magnetic field. Then we identify ITCs and asymptotic models for the electric field from those of the magnetic field. The conditions of order 2 turn out to be Poincaré-Steklov maps between tangential components of the magnetic field and the electric field. The new conditions of order 2 are simpler than the second order conditions for the time-harmonic Maxwell equations since the new Poincaré-Steklov operator is the scalar part of the Poincaré-Steklov operator of Wentzell type [27]. Hence it is worth noting that, contrary to [27], the discretization of the new second order condition does not require to introduce a mixed variational formulation including auxiliary unknowns and the new Poincaré-Steklov map can be incorporated in the weak sense in a simpler variational formulation. Finally, the conditions of order 1 are the perfect electric conductor (PEC) boundary conditions, and this is the only similarity with the work in Ref. [27].

This paper is organized as follows. Section 2 presents the model with a formulation in terms of the magnetic field and a formal derivation of impedance conditions based on an asymptotic expansion in the thickness parameter $\varepsilon$. Then, in Section 3 as main results the asymptotic (also called equivalent) model of order 1 and the equivalent model of order 2 are given. The equivalent model of order 2 for the electric field involves as an ITC a generalized Poincaré-Steklov map (tangential components of magnetic field to tangential components of electric field), whose structure simplifies for a symmetric configuration of material constants. We introduce at the end of Section 3 a regularized variational formulation for the second order model and we prove stability results for this model. The derivation of the equivalent models is based on an asymptotic expansion for the magnetic field which is presented in detail and order by order in Section 5 . Finally we introduce variational formulations for the exact electric field and for the limit model $\mathbf{E}_{0}$, and we prove stability and convergence results, Section 4. 
VICTOR PÉRON

\section{THE MATHEMATICAL MODEL AND ASYMPTOTIC MODELS WITH TRANSMISSION CONDITIONS}

After the introduction of notations in Sec. 2.1 and the mathematical model for the electric and magnetic field in Sec. 2.2 we present the magnetic field formulation in Sec. 2.3. Then we present a guideline on the derivation of asymptotic models and impedance conditions (section 2.4), where impedance conditions for the considered model will be given up to order 2 in the Sec. 3.

2.1. Notations. For any orientable and closed surface $\Gamma$ of $\mathbb{R}^{3}$ the unit normal vector $\mathbf{n}$ on $\Gamma$ is outwardly oriented from the interior domain enclosed by $\Gamma$ towards the outer domain, see e.g. Fig. 1. Let $\mathbf{v}$ a vector field on $\Gamma$, then we denote by

$$
\mathbf{v}_{\mathbf{T}}=\mathbf{n} \times(\mathbf{v} \times \mathbf{n}),
$$

the vector field of its tangent components and the space of $L^{2}$-integrable tangent vector fields by $\mathbf{L}_{t}^{2}(\Gamma):=\left\{\mathbf{v} \in\left(\mathrm{L}^{2}(\Gamma)\right)^{3}, \mathbf{v} \cdot \mathbf{n}=0\right.$ on $\left.\Gamma\right\}$.

We denote by $\operatorname{curl}_{\Gamma}$ the tangential rotational operator (which applies to functions defined on $\Gamma)$ and by $\operatorname{curl}_{\Gamma}$ the surface rotational operator (which applies to vector fields) $[26,10,27]$ :

$$
\begin{gathered}
\forall f \in C^{\infty}(\Gamma), \quad \operatorname{curl}_{\Gamma} f=\left(\nabla_{\Gamma} f\right) \times \mathbf{n}, \\
\forall \mathbf{v} \in\left(C^{\infty}(\Gamma)\right)^{3}, \quad \operatorname{curl}_{\Gamma} \mathbf{v}=\operatorname{div}_{\Gamma}(\mathbf{v} \times \mathbf{n}),
\end{gathered}
$$

where $\nabla_{\Gamma}$ and $\operatorname{div}_{\Gamma}$ are respectively the tangential gradient and the surface divergence on $\Gamma$.

Let $\Omega_{-}$and $\Omega_{+}$be Lipschitz domains with a common interface $\Gamma:=\partial \Omega_{-} \cap \partial \Omega_{+}$, which is a closed set, and let $\mathbf{n}$ on $\Gamma$ be the unit normal vector directed into $\Omega_{+}$(see Fig. 2). Then, for functions $f \in C^{\infty}\left(\Omega_{ \pm}\right)$, which are possibly discontinuous over the interface $\Gamma$, we denote by $[f]_{\Gamma}$ the jump of $f$ across $\Gamma$ :

$$
[f]_{\Gamma}=\left.f\right|_{\Gamma^{+}}-\left.f\right|_{\Gamma^{-}}
$$

where for any $\mathbf{x}_{\Gamma} \in \Gamma$ the one-sided traces are defined by

$$
\left.f\right|_{\Gamma^{ \pm}}\left(\mathbf{x}_{\Gamma}\right):=\lim _{s \rightarrow 0^{ \pm}} f\left(\mathbf{x}_{\Gamma}+s \mathbf{n}\right) .
$$

Furthermore, we denote by $\{f\}_{\Gamma}$ the mean value of $f$ across $\Gamma$ :

$$
\{f\}_{\Gamma}=\frac{1}{2}\left(\left.f\right|_{\Gamma^{+}}+\left.f\right|_{\Gamma^{-}}\right) .
$$

We use the same definition for vector fields $\mathbf{v} \in\left(C^{\infty}\left(\Omega_{ \pm}\right)\right)^{3}$, and with an abuse of notation, for the tangential traces:

$$
\begin{array}{rlrl}
\{\mathbf{v} \times \mathbf{n}\}_{\Gamma} & :=\{\mathbf{v}\}_{\Gamma} \times \mathbf{n}, & {[\mathbf{v} \times \mathbf{n}]_{\Gamma}:=[\mathbf{v}]_{\Gamma} \times \mathbf{n}} \\
\left\{\mathbf{v}_{\mathbf{T}}\right\}_{\Gamma}:=\left(\{\mathbf{v}\}_{\Gamma}\right)_{\mathbf{T}}, & {\left[\mathbf{v}_{\mathbf{T}}\right]_{\Gamma}:=\left([\mathbf{v}]_{\Gamma}\right)_{\mathbf{T}}}
\end{array}
$$

2.2. The time-harmonic eddy current problem. Throughout the paper we denote by $\Omega \subset \mathbb{R}^{3}$ a simply connected domain. Let $\Omega_{-}^{\varepsilon}$ be a simply connected subdomain of $\Omega$ and let $\Omega_{o}^{\varepsilon}$ be a thin layer of constant thickness $\varepsilon$ surrounding $\Omega_{-}^{\varepsilon}$ such that $\Omega$ contains $\Omega_{-}^{\varepsilon} \cup \overline{\Omega_{o}^{\varepsilon}}$, see Figure 1. For the sake of simplicity we further assume that the boundaries $\partial \Omega$ and $\Gamma_{-}^{\varepsilon}=\partial \Omega_{-}^{\varepsilon}$ are connected. Now the exterior domain is defined as $\Omega_{+}^{\varepsilon}=\Omega \backslash\left(\Omega_{-}^{\varepsilon} \cup \overline{\Omega_{o}^{\varepsilon}}\right)$ and the boundary of the subdomain $\overline{\Omega_{-}^{\varepsilon}} \cup \Omega_{o}^{\varepsilon}$ is denoted by $\Gamma_{+}^{\varepsilon}$. In all that follows, unless specified, all the considered domains are smooth domains in $\mathbb{R}^{3}$. 


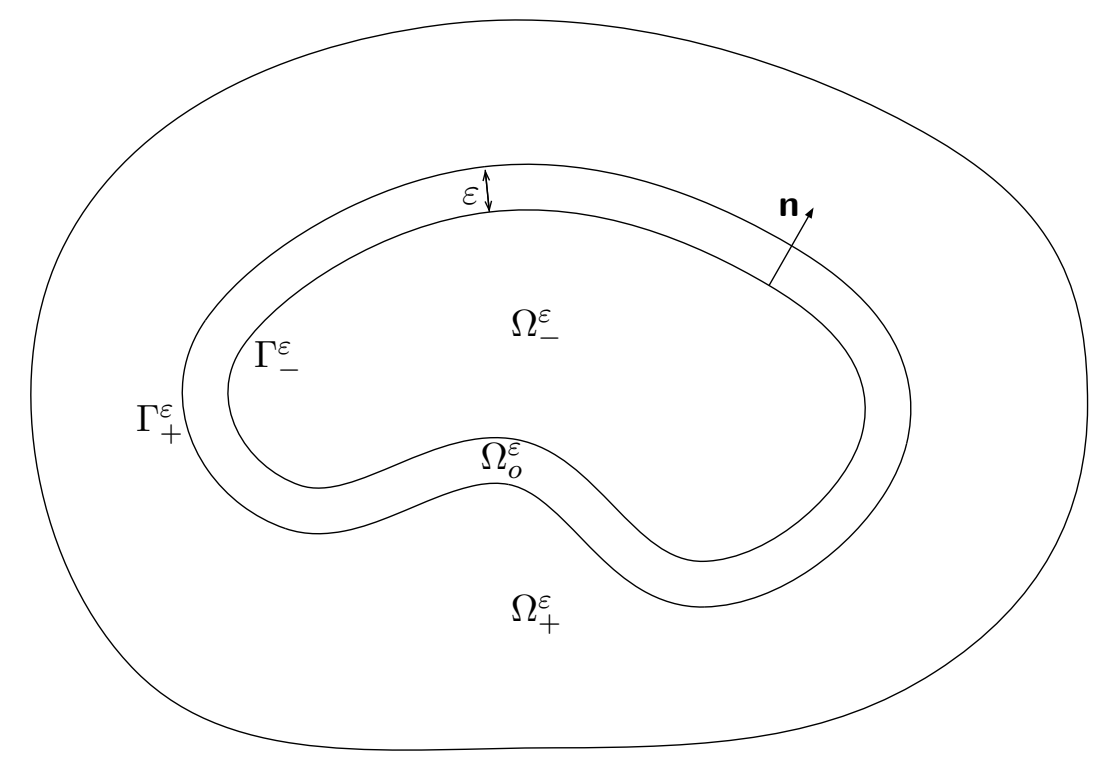

FIGURE 1. A cross-section of the domain $\Omega$ and the subdomains $\Omega_{-}^{\varepsilon}, \Omega_{o}^{\varepsilon}, \Omega_{+}^{\varepsilon}$.

The electromagnetic properties in $\Omega$ are given by the piecewise-constant functions $\mu^{\varepsilon}$, and $\underline{\sigma}^{\varepsilon}$ corresponding to the respective magnetic permeability, and conductivity of the possibly different materials in the three subdomains. They are given by

$$
\underline{\mu}^{\varepsilon}=\left\{\begin{array}{ll}
\mu_{-}>0, & \text { in } \Omega_{-}^{\varepsilon}, \\
\mu_{o}>0, & \text { in } \Omega_{o}^{\varepsilon}, \\
\mu_{+}>0, & \text { in } \Omega_{+}^{\varepsilon},
\end{array} \quad \underline{\sigma}^{\varepsilon}= \begin{cases}0, & \text { in } \Omega_{-}^{\varepsilon}, \\
\sigma_{o}^{\varepsilon}=\varepsilon^{-2} \tilde{\sigma}>0, & \text { in } \Omega_{o}^{\varepsilon}, \\
0, & \text { in } \Omega_{+}^{\varepsilon} .\end{cases}\right.
$$

We consider $\varepsilon$ as a parameter, on which $\mu^{\varepsilon}$ depend through the definition of the subdomains, where in $\underline{\sigma}^{\varepsilon}$ in addition we assume an explicit dependence of the layer conductivity $\sigma_{o}^{\varepsilon}$ on $\varepsilon$. With this correlation the thinner is the layer, the larger is the conductivity in the layer. The dependence like $\varepsilon^{-2}$ corresponds for $\varepsilon \rightarrow 0$ to asymptotically constant ratio of skin depth $d_{\text {skin }}=$ $\sqrt{2 /\left(\omega \mu_{o} \sigma_{o}^{\varepsilon}\right)}$ and thickness $\varepsilon[30,27]$, i.e., they behave the same for $\varepsilon \rightarrow 0$.

Let us denote by $\mathbf{j}$ the time-harmonic current source (with time convention $\exp (-\mathrm{i} \omega t)$ ) and let $\omega>0$ be the angular frequency. For the sake of simplicity, we assume that $\mathbf{j}$ is smooth enough, $\mathbf{j}$ is divergence free $\left(\operatorname{div} \mathbf{j}=0\right.$ in $\Omega$ ) and the support of $\mathbf{j}$ does not meet the layer $\Omega_{o}^{\varepsilon}$, and we write $\mathbf{j}_{ \pm}=\mathbf{j}$ in $\Omega_{ \pm}^{\varepsilon}$. The time-harmonic eddy current problem is $[6,14,9]$ :

$$
\begin{array}{r}
\operatorname{curl} \mathbf{E}^{\varepsilon}-i \omega \underline{\mu}^{\varepsilon} \mathbf{H}^{\varepsilon}=0 \quad \text { and } \operatorname{curl} \mathbf{H}^{\varepsilon}-\underline{\sigma}^{\varepsilon} \mathbf{E}^{\varepsilon}=\mathbf{j} \quad \text { in } \Omega, \\
\operatorname{div} \mathbf{E}_{ \pm}^{\varepsilon}=0 \text { in } \Omega_{-}^{\varepsilon} \cup \Omega_{+}^{\varepsilon} \quad \text { and } \int_{\Gamma_{ \pm}^{\varepsilon}} \mathbf{E}_{ \pm}^{\varepsilon} \cdot \mathbf{n} \mathrm{d} S=0 .
\end{array}
$$

The equations (2.1a) are Faraday's and Ampère's laws without displacement current. These equations link the electric field $\mathbf{E}^{\varepsilon}$ and the magnetic field $\mathbf{H}^{\varepsilon}$. The equations (2.1b) are gauge conditions for the electric field, we refer the reader to Remark 3 in [9] for a justification of 
such conditions. We complement this problem with a perfect electric conductor (PEC) boundary condition

$$
\mathbf{E}^{\varepsilon} \times \mathbf{n}=0 \quad \text { and } \quad \mathbf{H}^{\varepsilon} \cdot \mathbf{n}=0 \quad \text { on } \quad \partial \Omega .
$$

Remark 2.1. (i) Condition $\int_{\Gamma^{\varepsilon}} \mathbf{E}_{-}^{\varepsilon} \cdot \mathbf{n} \mathrm{d} S=0$ in (2.1b) is not necessary since it is a consequence of the divergence-free condition $\operatorname{div} \mathbf{E}_{-}^{\varepsilon}=0$ in $\Omega_{-}^{\varepsilon}$.

(ii) Boundary condition $\mathbf{H}^{\varepsilon} \cdot \mathbf{n}=0$ on $\partial \Omega$ is not needed, as it is a consequence of the condition $\mathbf{E}^{\varepsilon} \times \mathbf{n}=0$ on $\partial \Omega$.

2.3. Magnetic field formulation. The system of first order partial differential equations (2.1) can be reduced to a system of second order partial differential equations for the magnetic field by eliminating the electric field $[5,1]$

$$
\begin{aligned}
\operatorname{curl} \operatorname{curl} \mathbf{H}_{o}^{\varepsilon}-\mathrm{i} \omega \mu_{o} \sigma_{o}^{\varepsilon} \mathbf{H}_{o}^{\varepsilon} & =0, & & \text { in } \Omega_{o}^{\varepsilon}, \\
\operatorname{curl} \mathbf{H}_{ \pm}^{\varepsilon} & =\mathbf{j}_{ \pm}, & & \text {in } \Omega_{-}^{\varepsilon} \cup \Omega_{+}^{\varepsilon}, \\
\operatorname{div} \mathbf{H}_{ \pm}^{\varepsilon} & =0, & & \text { in } \Omega_{-}^{\varepsilon} \cup \Omega_{+}^{\varepsilon},
\end{aligned}
$$

with the transmission conditions across the two conductor surfaces $\Gamma_{+}^{\varepsilon}$ and $\Gamma_{-}^{\varepsilon}$

$$
\begin{aligned}
\mathbf{H}_{ \pm}^{\varepsilon} \times \mathbf{n} & =\mathbf{H}_{o}^{\varepsilon} \times \mathbf{n}, & & \text { on } \quad \Gamma_{ \pm}^{\varepsilon}, \\
\mu_{ \pm} \mathbf{H}_{ \pm}^{\varepsilon} \cdot \mathbf{n} & =\mu_{o} \mathbf{H}_{o}^{\varepsilon} \cdot \mathbf{n}, & & \text { on } \Gamma_{ \pm}^{\varepsilon},
\end{aligned}
$$

and with the perfect magnetic conductor (PMC) boundary condition

$$
\mathbf{H}^{\varepsilon} \cdot \mathbf{n}=0 \quad \text { on } \partial \Omega
$$

Here $\mathbf{H}_{\dagger}^{\varepsilon}, \dagger=-, o,+$ denote the restrictions of $\mathbf{H}^{\varepsilon}$ to the respective subdomain $\Omega_{\dagger}^{\varepsilon}$.

2.4. Guideline on the derivation of equivalent models and impedance conditions. In this section we give a guideline on the derivation of impedance conditions for the magnetic field (2.2), which is based on an asymptotic expansion in the thickness parameter $\varepsilon$ in Sect. 5. We will then propose two equivalent models $\mathbf{H}_{0}$ (in Sec. 3.1) and $\mathbf{H}_{\varepsilon}^{1}$ (in Sec. 3.2) for the magnetic field. The first model $\mathbf{H}_{0}$ is of order 1 , i.e., it satisfies at least formally $\mathbf{H}^{\varepsilon}-\mathbf{H}_{0}=\mathcal{O}(\varepsilon)$ and the second model $\mathbf{H}_{\varepsilon}^{1}$ is of order 2, i.e. it satisfies at least formally $\mathbf{H}^{\varepsilon}-\mathbf{H}_{\varepsilon}^{1}=\mathcal{O}\left(\varepsilon^{2}\right)$. These models are defined in $\varepsilon$-independent domains $\Omega_{-}, \Omega_{+}$, where $\Omega_{-}$denotes the domain $\Omega_{-}^{\varepsilon}$ in the limit $\varepsilon \rightarrow 0$ and $\Omega_{+}$the domain $\Omega_{+}^{\varepsilon}$ for $\varepsilon \rightarrow 0$, i.e. $\Omega_{+}=\Omega \backslash \bar{\Omega}_{-}$(see Figure 2). The surface $\Gamma$ (which is the mid-surface of the thin layer $\Omega_{o}^{\varepsilon}$ ) denotes the boundary of $\Omega_{-}$.

To define these equivalent models it is convenient to introduce the electromagnetic properties of the "background" problem by simple extension of the values of $\underline{\mu}^{\varepsilon}$ outside the sheet in the extended domains $\Omega_{-}$and $\Omega_{+}$:

$$
\mu= \begin{cases}\mu_{-}, & \text {in } \Omega_{-}, \\ \mu_{+}, & \text {in } \Omega_{+} .\end{cases}
$$

In the following we present briefly a formal derivation of impedance conditions. We summarize this process in two steps. 


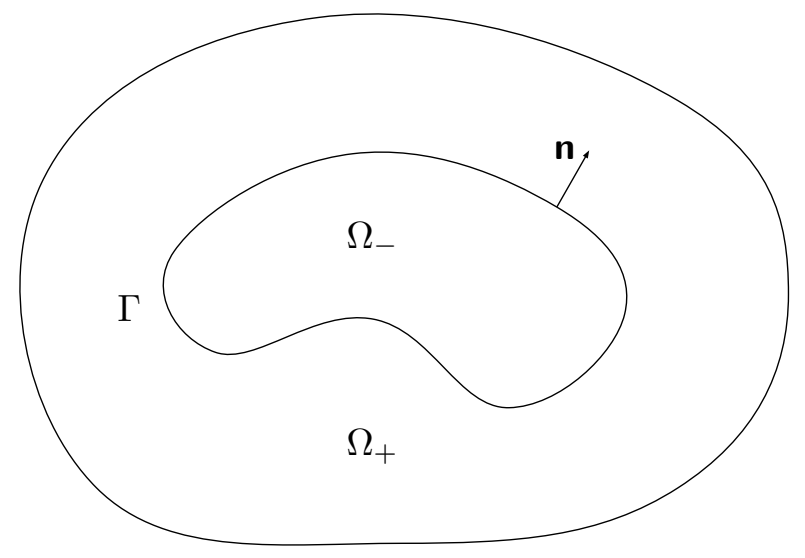

FIGURE 2. A cross-section of the domain $\Omega$ and the subdomains $\Omega_{-}, \Omega_{+}$.

First step : a multiscale expansion. The first step consists in deriving a multiscale expansion for the solution $\mathbf{H}^{\varepsilon}$ of the model problem (2.2) : it possesses an asymptotic expansion in power series of the small parameter $\varepsilon$

$$
\begin{array}{ll}
\mathbf{H}^{\varepsilon}(\mathbf{x}) \approx \mathbf{H}_{0}(\mathbf{x})+\varepsilon \mathbf{H}_{1}(\mathbf{x})+\varepsilon^{2} \mathbf{H}_{2}(\mathbf{x})+\cdots \quad \text { for a.e. } \quad \mathbf{x} \in \Omega_{-}^{\varepsilon} \cup \Omega_{+}^{\varepsilon}, \\
\mathbf{H}^{\varepsilon}(\mathbf{x}) \approx \underline{\mathfrak{Y}}_{0}\left(y_{\alpha}, \frac{h}{\varepsilon}\right)+\varepsilon \underline{\mathfrak{Y}}_{1}\left(y_{\alpha}, \frac{h}{\varepsilon}\right)+\cdots \quad \text { for a.e. } \quad \mathbf{x} \in \Omega_{o}^{\varepsilon} .
\end{array}
$$

Here, $\mathbf{x} \in \mathbb{R}^{3}$ are the cartesian coordinates, $\left(y_{\alpha}, h\right)$ is a local normal coordinate system [7] to the surface $\Gamma$ in the thin layer $\Omega_{o}^{\varepsilon}$ where $y_{\alpha}, \alpha=1,2$ are tangential coordinates on $\Gamma$ and $h \in\left(-\frac{\varepsilon}{2}, \frac{\varepsilon}{2}\right)$ is the normal coordinate to $\Gamma$. Moreover, the term $\underline{\mathfrak{H}}_{j}$ is a "profile" defined on $\Gamma \times\left(-\frac{1}{2}, \frac{1}{2}\right)$. Note, that the intrinsic domain of the "far field terms" $\mathbf{H}_{j}$ is $\Omega_{-} \cup \Omega_{+}$. The first terms $\left(\underline{\mathfrak{Y}}_{j}, \mathbf{H}_{j}\right)$ for $j=0,1$ are derived step by step in Section 5 .

The derivation is based on an expansion of the differential operators inside the thin layer $\Omega_{o}^{\varepsilon}$ in terms of $\varepsilon$, a Taylor expansion of $\left.\mathbf{H}_{j}\right|_{\Gamma_{ \pm}^{\varepsilon}}$ around the mid-surface $\Gamma$ and a collection of terms of same powers of $\varepsilon$ in the governing PDE inside and outside the sheet, the transmission conditions for the traces on $\Gamma_{ \pm}^{\varepsilon}$ and the boundary conditions. Since, moreover, the terms $\underline{\mathfrak{H}}_{j}$ of the expansion inside the sheet can be explicitly expressed in terms of $\mathbf{H}_{i}, i=0, \ldots, j-1$ we obtain formally

$$
\begin{aligned}
\operatorname{curl} \mathbf{H}_{j}^{ \pm} & =\mathbf{j}^{ \pm} \delta_{0 j}, \quad \text { in } \Omega_{ \pm}, \\
\operatorname{div} \mathbf{H}_{j}^{ \pm} & =0, \quad \text { in } \Omega_{ \pm}, \\
\mathbf{H}_{j}^{+} \cdot \mathbf{n} & =0, \quad \text { on } \partial \Omega,
\end{aligned}
$$

for the restrictions $\mathbf{H}_{j}^{ \pm}$of $\mathbf{H}_{j}$ to $\Omega_{ \pm}$, with boundary conditions on $\Gamma$ :

$$
\mu_{ \pm} \mathbf{H}_{j}^{ \pm} \cdot \mathbf{n}=\sum_{i=0}^{j} \mathcal{G}_{i}^{ \pm}\left(\begin{array}{c}
\left\{\left(\mathbf{H}_{j-i}\right)_{\mathbf{T}}\right\}_{\Gamma} \\
{\left[\left(\mathbf{H}_{j-i}\right)_{\mathbf{T}}\right]_{\Gamma}}
\end{array}\right) .
$$


Here, $\delta_{0 j}=1$ if $j=0$ and zero otherwise and $\mathcal{G}_{i}^{ \pm}$in the discrete convolution on the right hand side of (2.4d) are differential operators on $\Gamma$ not depending on $\varepsilon$ where $\mathcal{G}_{0}^{ \pm}=0$.

Second step : construction of impedance conditions and equivalent models. The second step consists in identifying a simpler problem satisfied by an approximation $\mathbf{H}_{\varepsilon}^{k}$ of the truncated expansion $\mathbf{H}_{0}(\mathbf{x})+\varepsilon \mathbf{H}_{1}(\mathbf{x})+\varepsilon^{2} \mathbf{H}_{2}(\mathbf{x})+\cdots+\varepsilon^{k} \mathbf{H}_{k}(\mathbf{x})$ up to a residual term in $\mathcal{O}\left(\varepsilon^{k+1}\right)$. For this the equations in (2.4) for $i=0, \ldots, k$ are multiplied with $\varepsilon^{i}$ and added up, and terms in $\mathcal{O}\left(\varepsilon^{k+1}\right)$ are neglected. In this way we obtain the simpler problem as

$$
\begin{array}{rc}
\operatorname{curl} \mathbf{H}_{\varepsilon}^{k}=\mathbf{j}, & \text { in } \Omega_{-} \cup \Omega_{+}, \\
\operatorname{div} \mathbf{H}_{\varepsilon}^{k}=0, & \text { in } \Omega_{-} \cup \Omega_{+}, \\
\mathbf{H}_{\varepsilon}^{k} \cdot \mathbf{n}=0, & \text { on } \partial \Omega,
\end{array}
$$

with the following transmission conditions on $\Gamma$ :

$$
\left(\begin{array}{c}
{\left[\mu \mathbf{H}_{\varepsilon}^{k} \cdot \mathbf{n}\right]_{\Gamma}} \\
\left\{\mu \mathbf{H}_{\varepsilon}^{k} \cdot \mathbf{n}\right\}_{\Gamma}
\end{array}\right)=\mathcal{G}_{k, \varepsilon}\left(\begin{array}{c}
\left\{\left(\mathbf{H}_{\varepsilon}^{k}\right)_{\mathbf{T}}\right\}_{\Gamma} \\
{\left[\left(\mathbf{H}_{\varepsilon}^{k}\right)_{\mathbf{T}}\right]_{\Gamma}}
\end{array}\right),
$$

where $\mathcal{G}_{k, \varepsilon}=\sum_{i=0}^{k} \varepsilon^{i}\left(\left[\mathcal{G}_{i}\right]_{\Gamma},\left\{\mathcal{G}_{i}\right\}_{\Gamma}\right)^{\top}$ is the truncation of the weigthed sum of operators $\mathcal{G}_{i}^{ \pm}$ where the jump or mean value is taken respectively. With this derivation it holds at least formally $\mathbf{H}^{\varepsilon}-\mathbf{H}_{\varepsilon}^{k}=\mathcal{O}\left(\varepsilon^{k+1}\right)$. Hence, we say that $\mathbf{H}_{\varepsilon}^{k}$ is an equivalent (or approximate) model of order $k+$ 1.

In this paper, we give explicitly the equivalent models of order 1 and 2 in Section 3. Their derivations are presented in detail in Section 5.

\section{MAIN RESUlts. ASYMPTOTIC MODELS UP TO ORDER 2}

In this section we present the main results of the paper. We introduce the approximate models of order 1 (Section 3.1) and 2 (Section 3.2) for the magnetic field. Then we deduce equivalent models up to the second order of approximation for the electric field (Section 3.3). Finally we introduce a regularized variational formulation for the second order model (Section 3.4) and we prove well-posedness and stability results for this model.

3.1. Equivalent model of order 1 for the magnetic field. The equivalent model of order 1 is given by the limit solution $\mathbf{H}_{0}$ of (2.2) when $\varepsilon \rightarrow 0$. The limit solution satisfies the perfectly conducting magnetic (PMC) boundary condition on $\Gamma$ and can be defined independently in the two subdomains $\Omega_{-}, \Omega_{+}$. Hence, $\mathbf{H}_{0}^{-}=\left.\mathbf{H}_{0}\right|_{\Omega_{-}}$is a tangential vector potential which satisfies

$$
\begin{aligned}
\operatorname{curl} \mathbf{H}_{0}^{-} & =\mathbf{j}_{-}, & & \text {in } \Omega_{-}, \\
\operatorname{div} \mathbf{H}_{0}^{-} & =0, & & \text { in } \Omega_{-}, \\
\mathbf{H}_{0}^{-} \cdot \mathbf{n} & =0, & & \text { on } \Gamma,
\end{aligned}
$$


whereas $\mathbf{H}_{0}^{+}=\left.\mathbf{H}_{0}\right|_{\Omega_{+}}$is a tangential vector potential given by

$$
\begin{aligned}
\operatorname{curl} \mathbf{H}_{0}^{+} & =\mathbf{j}_{+}, & & \text {in } \Omega_{+}, \\
\operatorname{div} \mathbf{H}_{0}^{+} & =0, & & \text { in } \Omega_{+}, \\
\mathbf{H}_{0}^{+} \cdot \mathbf{n} & =0, & & \text { on } \Gamma, \\
\mathbf{H}_{0}^{+} \cdot \mathbf{n} & =0, & & \text { on } \partial \Omega .
\end{aligned}
$$

The boundary condition on $\partial \Omega$ is not affected by the limiting process $\varepsilon \rightarrow 0$ and transfers simply to the limit solution $\mathbf{H}_{0}$.

The next proposition ensures that the vector potentials satisfying problems (3.1) and (3.2) are well-defined in the framework above and even in Lipschitz domains. We recall the definition of the classical spaces

$$
X_{\mathbf{T}}\left(\Omega_{-}\right)=\left\{\mathbf{u} \in \mathbf{H}\left(\operatorname{curl}, \Omega_{-}\right) \mid \operatorname{div} \mathbf{u} \in \mathrm{L}^{2}\left(\Omega_{-}\right), \quad \mathbf{u} \cdot \mathbf{n}=0 \quad \text { on } \Gamma\right\},
$$

and

$$
X_{\mathbf{T}}\left(\Omega_{+}\right)=\left\{\mathbf{u} \in \mathbf{H}\left(\operatorname{curl}, \Omega_{+}\right) \mid \operatorname{div} \mathbf{u} \in \mathrm{L}^{2}\left(\Omega_{+}\right), \quad \mathbf{u} \cdot \mathbf{n}=0 \quad \text { on } \Gamma \cup \partial \Omega\right\} .
$$

Hereafter the symbol $\langle\cdot, \cdot\rangle_{\Gamma}$ denotes the duality pairing between $\mathrm{H}^{-1 / 2}(\Gamma)$ and $\mathrm{H}^{1 / 2}(\Gamma)$.

Proposition 3.1. Let $\mathbf{j} \in \mathbf{L}^{2}(\Omega)$ such that $\operatorname{div} \mathbf{j}^{ \pm}=0$ in $\Omega_{ \pm}$and $\left\langle\mathbf{j}^{+} \cdot \mathbf{n}, 1\right\rangle_{\Gamma}=0$. Then there exists a unique vector potential $\mathbf{H}_{0}^{-} \in \mathbf{X}_{\mathbf{T}}\left(\Omega_{-}\right)$satisfying (3.1) and there exists a unique vector potential $\mathbf{H}_{0}^{+} \in \mathbf{X}_{\mathbf{T}}\left(\Omega_{+}\right)$satisfying (3.2). Furthermore the magnetic field $\mathbf{H}_{0}^{+}$belongs to $\mathbf{H}^{1}\left(\Omega_{+}\right)$ and $\mathbf{H}_{0}^{-}$belongs to $\mathbf{H}^{1}\left(\Omega_{-}\right)$.

Remark 3.2. In the framework of Section 2.2 the right-hand side $\mathbf{j}$ of the eddy current problem (2.1) satisfies all the assumptions in Proposition 3.1.

Proof. We recall that the domains $\Omega_{ \pm}$are simply connected, the surface $\partial \Omega_{+}$has two connected components $(\Gamma$ and $\partial \Omega)$ and the surface $\partial \Omega_{-}$is connected. Then the proof of existence and uniqueness of tangential vector potentials $\mathbf{H}_{0}^{ \pm} \in \mathbf{X}_{\mathbf{T}}\left(\Omega_{ \pm}\right)$can be found in [2, Th 3.12] for Lipschitz domains in $\mathbb{R}^{3}$. The proof of the regularity result can be found in [2, Th 2.9] when the domains $\Omega_{ \pm}$are of class $\mathcal{C}^{1,1}$ (see also [13, chapter I, section 3.5]). We refer the reader to the reference [11] for the proof of this regularity result when the domains $\Omega_{ \pm}$are smooths.

3.2. Equivalent model of order 2 for the magnetic field. We define a second order approximate solution $\mathbf{H}_{\varepsilon}^{1}$, which shall be much more accurate approximation of $\mathbf{H}^{\varepsilon}$ than the limit solution $\mathbf{H}_{0}$ when $\varepsilon \rightarrow 0$. The equations defining $\mathbf{H}_{\varepsilon}^{1}$ outside the mid-surface $\Gamma$ remain the same, i.e., it solves

$$
\begin{array}{rc}
\operatorname{curl} \mathbf{H}_{\varepsilon}^{1}=\mathbf{j}, & \text { in } \Omega_{-} \cup \Omega_{+}, \\
\operatorname{div} \mathbf{H}_{\varepsilon}^{1}=0, & \text { in } \Omega_{-} \cup \Omega_{+}, \\
\mathbf{H}_{\varepsilon}^{1} \cdot \mathbf{n}=0, & \text { on } \partial \Omega,
\end{array}
$$

and at the mid-surface $\Gamma$ the transmission conditions

$$
\left(\begin{array}{c}
{\left[\mu \mathbf{H}_{\varepsilon}^{1} \cdot \mathbf{n}\right]_{\Gamma}} \\
\left\{\mu \mathbf{H}_{\varepsilon}^{1} \cdot \mathbf{n}\right\}_{\Gamma}
\end{array}\right)=\varepsilon\left(\begin{array}{cc}
\mathrm{L}_{1} & \mathrm{~L}_{3} \\
\mathrm{~L}_{3} & \mathrm{~L}_{2}
\end{array}\right)\left(\begin{array}{c}
\left\{\left(\mathbf{H}_{\varepsilon}^{1}\right)_{\mathbf{T}}\right\}_{\Gamma} \\
{\left[\left(\mathbf{H}_{\varepsilon}^{1}\right)_{\mathbf{T}}\right]_{\Gamma}}
\end{array}\right)
$$


are posed. Here, $[\cdot]_{\Gamma}$ and $\{\cdot\}_{\Gamma}$ denote the jump and averages introduced in Sec. 2.1 and $\mathrm{L}_{i}$ are first order differential operators given by

$$
\mathrm{L}_{i}=C_{i} \operatorname{div}_{\Gamma}, \quad i=1,2,3,
$$

in which $C_{i}$ are constants defined by

$$
\begin{aligned}
C_{1} & =\{\mu\}_{\Gamma}-2 \frac{\mu_{o}}{\gamma} \tanh \left(\frac{\gamma}{2}\right), \\
C_{2} & =\frac{\{\mu\}_{\Gamma}}{4}-\frac{\mu_{o}}{2 \gamma} \operatorname{coth}\left(\frac{\gamma}{2}\right), \\
C_{3} & =\frac{1}{4}[\mu]_{\Gamma},
\end{aligned}
$$

and

$$
\gamma=\exp \left(\frac{3 \mathrm{i} \pi}{4}\right) \sqrt{\omega \mu_{o} \tilde{\sigma}}
$$

Equivalent model of order 2 in a "symmetric" configuration. If the electromagnetic properties on both sides of the sheet are the same, i.e., $\mu_{+}=\mu_{-}=: \mu$ in $\Omega_{+} \cup \Omega_{-}$, then the transmission conditions (3.3d) of the second order model simplify to

$$
\begin{aligned}
{\left[\mu \mathbf{H}_{\varepsilon}^{1} \cdot \mathbf{n}\right]_{\Gamma} } & =\varepsilon C \operatorname{div}_{\Gamma}\left\{\left(\mathbf{H}_{\varepsilon}^{1}\right)_{\mathbf{T}}\right\}_{\Gamma}, \\
\left\{\mu \mathbf{H}_{\varepsilon}^{1} \cdot \mathbf{n}\right\}_{\Gamma} & =\varepsilon D \operatorname{div}_{\Gamma}\left[\left(\mathbf{H}_{\varepsilon}^{1}\right)_{\mathbf{T}}\right]_{\Gamma},
\end{aligned}
$$

where

$$
C=\mu-\frac{2 \mu_{o}}{\gamma} \tanh \left(\frac{\gamma}{2}\right), \quad D=\frac{\mu}{4}-\frac{\mu_{o}}{2 \gamma} \operatorname{coth}\left(\frac{\gamma}{2}\right)
$$

3.3. Equivalent models and impedance transmission conditions for the electric field. In this section we deduce a second order model for the electric field $\mathbf{E}_{\varepsilon}^{1}$ from the magnetic field $\mathbf{H}_{\varepsilon}^{1}$. Using the Faraday's law and the Stokes formula, there holds

$$
\left(\mathbf{H}_{\varepsilon}^{1, \pm}\right)_{\mathbf{T}}=\frac{1}{\mathrm{i} \omega \mu_{ \pm}}\left(\operatorname{curl} \mathbf{E}_{\varepsilon}^{1, \pm}\right)_{\mathbf{T}} \quad \text { and } \quad \mu_{ \pm} \mathbf{H}_{\varepsilon}^{1, \pm} \cdot \mathbf{n}=\frac{1}{\mathrm{i} \omega} \operatorname{div}_{\Gamma}\left(\mathbf{E}_{\varepsilon}^{1, \pm} \times \mathbf{n}\right) \quad \text { on } \quad \Gamma .
$$

Then using the transmission conditions (3.3d) we can identify the following transmission conditions for the electric field

$$
\left(\begin{array}{c}
{\left[\mathbf{E}_{\varepsilon}^{1} \times \mathbf{n}\right]_{\Gamma}} \\
\left\{\mathbf{E}_{\varepsilon}^{1} \times \mathbf{n}\right\}_{\Gamma}
\end{array}\right)=\varepsilon\left(\begin{array}{ll}
C_{1} & C_{3} \\
C_{3} & C_{2}
\end{array}\right)\left(\begin{array}{c}
\left\{\frac{1}{\mu}\left(\operatorname{curl} \mathbf{E}_{\varepsilon}^{1}\right)_{\mathbf{T}}\right\}_{\Gamma} \\
{\left[\frac{1}{\mu}\left(\operatorname{curl} \mathbf{E}_{\varepsilon}^{1}\right)_{\mathbf{T}}\right]_{\Gamma}}
\end{array}\right),
$$

where the constants $C_{i}$ are defined in (3.4). The transmission conditions (3.8) can be regarded as a Poincaré-Steklov map $\mathbf{H}_{\mathbf{T}}$-to- $\mathbf{E} \times \mathbf{n}$ which tends to the PEC boundary condition for $\varepsilon \rightarrow 0$. As a consequence the equivalent model of order 1 for the electric field $\mathbf{E}_{0}$ (4.2)-(4.3) (in Section 4) is defined independently in the two subdomains $\Omega_{ \pm}$. 
Finally the second order model $\mathbf{E}_{\varepsilon}^{1}$ solves

$$
\begin{array}{rlrl}
\operatorname{curl} \frac{1}{\mu} \operatorname{curl} \mathbf{E}_{\varepsilon}^{1, \pm} & =\mathrm{i} \omega \mathbf{j}^{ \pm} \quad \text { in } \quad \Omega_{ \pm}, \\
\operatorname{div} \mathbf{E}_{\varepsilon}^{1, \pm} & =0 \quad \text { in } \quad \Omega_{ \pm}, \\
\int_{\Gamma} \mathbf{E}_{\varepsilon}^{1, \pm} \cdot \mathbf{n} \mathrm{d} S & =0, & \\
\mathbf{E}_{\varepsilon}^{1} \times \mathbf{n} & =0 \quad \text { on } \partial \Omega,
\end{array}
$$

and the transmission conditions (3.8) at the mid-surface $\Gamma$.

Remark 3.3. The conditions (3.8) are simpler than the second order conditions for the timeharmonic Maxwell equations [27, Eq. (7c)] since the new Poincaré-Steklov map (3.8) is the scalar part of the Poincaré-Steklov map of Wentzell type [27, Eq. (7c)]. It is worth noting that conditions (3.8) are consistent with the second order conditions ITC-2-1 [30, Eq. (2.17)] which have been derived for the time-harmonic eddy current model in two dimensions and for a constant magnetic permeability.

3.4. Regularized variational formulation for the electric field in the second order model. Following Section 3.3, we can deduce a regularized variational formulation for the electric field $\mathbf{E}_{\varepsilon}^{1}$ : we search for $\mathbf{E}_{\varepsilon}^{1}$ in the Hilbert space

$$
\begin{aligned}
\mathbf{Y}= & \left\{\mathbf{E} \in \mathbf{L}^{2}(\Omega), \operatorname{curl} \mathbf{E}^{ \pm} \in \mathbf{L}^{2}\left(\Omega_{ \pm}\right), \operatorname{div} \mathbf{E}^{ \pm} \in \mathrm{L}^{2}\left(\Omega_{ \pm}\right), \mathbf{E}^{ \pm} \times \mathbf{n} \in \mathbf{L}_{t}^{2}(\Gamma),\right. \\
& \left.\int_{\Gamma} \mathbf{E}^{ \pm} \cdot \mathbf{n} \mathrm{d} S=0, \mathbf{E}^{+} \times \mathbf{n}=0 \text { on } \partial \Omega\right\}
\end{aligned}
$$

This space is equipped with the norm

$$
\begin{aligned}
\|\mathbf{u}\|_{\mathbf{Y}}^{2}=\|\mathbf{u}\|_{0, \Omega}^{2}+\left\|\operatorname{curl} \mathbf{u}^{+}\right\|_{0, \Omega_{+}}^{2}+\left\|\operatorname{curl} \mathbf{u}^{-}\right\|_{0, \Omega_{-}}^{2} \\
+\left\|\operatorname{div} \mathbf{u}^{+}\right\|_{0, \Omega_{+}}^{2}+\left\|\operatorname{div} \mathbf{u}^{-}\right\|_{0, \Omega_{-}}^{2}+\left\|\mathbf{u}^{+} \times \mathbf{n}\right\|_{0, \Gamma}^{2}+\left\|\mathbf{u}^{-} \times \mathbf{n}\right\|_{0, \Gamma}^{2} .
\end{aligned}
$$

Remark 3.4. According to Theorem 2 in [8], since $\Omega_{-}$is a bounded simply connected domain with connected Lipschitz boundary $\Gamma$, any vector field $\mathbf{E} \in \mathbf{Y}$ satisfies $\mathbf{E}^{-} \in \mathbf{H}^{\frac{1}{2}}\left(\Omega_{-}\right)$.

Variational problem for the electric field $\mathbf{E}_{\varepsilon}^{1}$. For all $\varepsilon>0$ we consider the variational problem : Find $\mathbf{E} \in \mathbf{Y}$ such that for all $\mathbf{v} \in \mathbf{Y}$,

$$
a_{1}^{\varepsilon}(\mathbf{E}, \mathbf{v})=\mathrm{i} \omega \int_{\Omega} \mathbf{j} \cdot \overline{\mathbf{v}} \mathrm{d} \mathbf{x} .
$$

Here the sesquilinear form (in its regularized version) $a_{1}^{\varepsilon}$ is defined as

$$
\begin{aligned}
& a_{1}^{\varepsilon}(\mathbf{u}, \mathbf{v})=\int_{\Omega_{-}} \frac{1}{\mu_{-}} \operatorname{curl} \mathbf{u}^{-} \cdot \operatorname{curl} \overline{\mathbf{v}^{-}} \mathrm{d} \mathbf{x}+\int_{\Omega_{+}} \frac{1}{\mu_{+}} \operatorname{curl} \mathbf{u}^{+} \cdot \operatorname{curl} \overline{\mathbf{v}^{+}} \mathrm{d} \mathbf{x}+\int_{\Omega_{-}} \operatorname{div} \mathbf{u}^{-} \operatorname{div} \overline{\mathbf{v}^{-}} \mathrm{d} \mathbf{x} \\
& +\int_{\Omega_{+}} \operatorname{div} \mathbf{u}^{+} \operatorname{div} \overline{\mathbf{v}^{+}} \mathrm{d} \mathbf{x}-\varepsilon^{-1} \int_{\Gamma} \mathrm{A}^{-1}\left(\begin{array}{c}
\mathbf{u} \times \mathbf{n}] \\
\{\mathbf{u} \times \mathbf{n}\}
\end{array}\right) \cdot\left(\begin{array}{l}
{[\overline{\mathbf{v}} \times \mathbf{n}]} \\
\{\overline{\mathbf{v}} \times \mathbf{n}\}
\end{array}\right) \mathrm{d} S,
\end{aligned}
$$


and $A$ is the nonsingular matrix given by

$$
\mathrm{A}=\left(\begin{array}{ll}
C_{1} & C_{3} \\
C_{3} & C_{2}
\end{array}\right)
$$

where the constants $C_{i}$ are defined in (3.4).

Remark 3.5. Here we assume that $\operatorname{det} \mathrm{A} \neq 0$. Straightforward calculi lead to

$$
\operatorname{det} \mathrm{A}=\frac{\mu_{+} \mu_{-}}{4}-\{\mu\}_{\Gamma} \operatorname{coth}(\gamma) \frac{\mu_{o}}{\gamma}+\frac{\mu_{o}^{2}}{\gamma^{2}}
$$

and we have

$$
\mathrm{A}^{-1}=(\operatorname{det} \mathrm{A})^{-1}\left(\begin{array}{cc}
C_{2} & -C_{3} \\
-C_{3} & C_{1}
\end{array}\right)
$$

where the constants $C_{i}$ are defined in (3.4).

Lemma 3.6. We recall that the domains $\Omega_{ \pm}$are simply connected, $\partial \Omega_{-}=\Gamma$ is connected and $\partial \Omega_{+}=\Gamma \cup \partial \Omega$ has two connected components. Assume that the domains $\Omega_{ \pm}$are of class $\mathcal{C}^{1,1}$. Let the positive constants $\mu_{ \pm}, \mu_{o}, \tilde{\sigma}$ and $\omega$ be fixed such that $\operatorname{det} A \neq 0$ and assume that there exists $\beta \in\{z \in \mathbb{C} \mid \operatorname{Re} z>0\}$ such that

$\forall \mathbf{u} \in \mathbf{Y}, \quad-\operatorname{Re}\left(\beta \int_{\Gamma} \mathrm{A}^{-1}\left(\begin{array}{c}{[\mathbf{u} \times \mathbf{n}]} \\ \{\mathbf{u} \times \mathbf{n}\}\end{array}\right) \cdot\left(\begin{array}{c}{[\overline{\mathbf{u}} \times \mathbf{n}]} \\ \{\overline{\mathbf{u}} \times \mathbf{n}\}\end{array}\right) \mathrm{d} S\right) \gtrsim\left\|\mathbf{u}^{+} \times \mathbf{n}\right\|_{0, \Gamma}^{2}+\left\|\mathbf{u}^{-} \times \mathbf{n}\right\|_{0, \Gamma}^{2} \cdot$

Then there exists $\varepsilon_{0}>0$ such that for all $\varepsilon \in\left(0, \varepsilon_{0}\right)$, a $a_{1}^{\varepsilon}$ is strongly coercive on $\mathbf{Y}:$ there exists $c_{0}>0$ such that for all $\varepsilon \in\left(0, \varepsilon_{0}\right)$ and for all $\mathbf{u} \in \mathbf{Y}$

$$
\operatorname{Re}\left(\beta a_{1}^{\varepsilon}(\mathbf{u}, \mathbf{u})\right) \geqslant c_{0}\|\mathbf{u}\|_{\mathbf{Y}}^{2} .
$$

In (3.13), the notation $a \lesssim b$ means the existence of a constant $C>0$ which is independent of quantities $a, b$ and of the parameter $\varepsilon$, such that $a \leqslant C b$.

Remark 3.7. Assumption (3.13) is satisfied in the "symmetric" configuration (i.e., $\mu_{+}=\mu_{-}=: \mu$ in $\Omega_{+} \cup \Omega_{-}$) when $|\gamma|$ is small enough and $\mu_{o}>\mu$ since in this framework we can check the following inequality

$$
-\operatorname{Re}\left(\beta \int_{\Gamma} \mathrm{A}^{-1}\left(\begin{array}{l}
\mathrm{u} \\
\mathrm{v}
\end{array}\right) \cdot\left(\begin{array}{l}
\overline{\mathrm{u}} \\
\overline{\mathrm{v}}
\end{array}\right) \mathrm{d} S\right) \gtrsim\|\mathrm{u}\|_{0, \Gamma}^{2}+\|\mathrm{v}\|_{0, \Gamma}^{2},
$$

for all $\beta \in\{z \in \mathbb{C} \mid \operatorname{Re} z>0\}$ such that $\frac{\mu}{4} \operatorname{Re} \beta+\frac{1}{\omega \tilde{\sigma}} \operatorname{Im} \beta \leqslant 0$.

We will prove Lemma 3.6 in Section 3.5. As a consequence of Lemma 3.6 we deduce the following result as an application of the Lax-Milgram lemma.

Theorem 3.8. Assume that the domains $\Omega_{ \pm}$are of class $\mathcal{C}^{1,1}$. Let $\mathbf{j} \in \mathbf{L}^{2}(\Omega)$ satisfy $\operatorname{div} \mathbf{j}=0$ in $\Omega$ and $\mathbf{j} \cdot \mathbf{n}=0$ on $\Gamma_{ \pm}$. Let the positive constants $\mu_{ \pm}, \mu_{o}, \tilde{\sigma}$ and $\omega$ be fixed such that $\operatorname{det} \mathrm{A} \neq 0$ and assume that (3.13) holds.

Then there exists $\varepsilon_{0}>0$ such that for all $\varepsilon \in\left(0, \varepsilon_{0}\right)$ there exists a unique solution $\mathbf{E}_{\varepsilon}^{1} \in \mathbf{Y}$ to the variational problem (3.11) and we have uniform estimates

$$
\left\|\mathbf{E}_{\varepsilon}^{1}\right\|_{\mathbf{Y}} \leqslant C\|\mathbf{j}\|_{0, \Omega} .
$$


3.5. Proof of Lemma 3.6. Let $\mathbf{u}$ be any function in the space $\mathbf{Y}$. The proof of this Lemma involved a Helmholtz decomposition for $\mathbf{u}^{+}$(Section 3.5.1) and a compactness argument based on a regularity result for $\mathbf{u}^{-}$.

Proof of Lemma 3.6. Using Assumption (3.13), one notes that we have the following inequality

$$
\begin{aligned}
\operatorname{Re}\left(\beta a_{1}^{\varepsilon}(\mathbf{u}, \mathbf{u})\right) \gtrsim\left\|\operatorname{curl} \mathbf{u}^{+}\right\|_{0, \Omega_{+}}^{2}+\left\|\operatorname{curl} \mathbf{u}^{-}\right\|_{0, \Omega_{-}}^{2} & +\left\|\operatorname{div} \mathbf{u}^{+}\right\|_{0, \Omega_{+}}^{2}+\left\|\operatorname{div} \mathbf{u}^{-}\right\|_{0, \Omega_{-}}^{2} \\
& +\varepsilon^{-1}\left(\left\|\mathbf{u}^{+} \times \mathbf{n}\right\|_{0, \Gamma}^{2}+\left\|\mathbf{u}^{-} \times \mathbf{n}\right\|_{0, \Gamma}^{2}\right) .
\end{aligned}
$$

Thus there exists $\varepsilon_{0}>0$ such that for all $\varepsilon \in\left(0, \varepsilon_{0}\right)$ and for all $\mathbf{u} \in \mathbf{Y}$

$$
\begin{array}{r}
\operatorname{Re}\left(\beta a_{1}^{\varepsilon}(\mathbf{u}, \mathbf{u})\right) \gtrsim\left\|\operatorname{curl} \mathbf{u}^{+}\right\|_{0, \Omega_{+}}^{2}+\left\|\operatorname{curl} \mathbf{u}^{-}\right\|_{0, \Omega_{-}}^{2}+\left\|\operatorname{div} \mathbf{u}^{+}\right\|_{0, \Omega_{+}}^{2}+\left\|\operatorname{div} \mathbf{u}^{-}\right\|_{0, \Omega_{-}}^{2} \\
+\left\|\mathbf{u}^{+} \times \mathbf{n}\right\|_{0, \Gamma}^{2}+\left\|\mathbf{u}^{-} \times \mathbf{n}\right\|_{0, \Gamma}^{2} .
\end{array}
$$

It remains to prove that the right hand-side above is an upper bound for $\|\mathbf{u}\|_{0, \Omega}^{2}=\left\|\mathbf{u}^{-}\right\|_{0, \Omega_{-}}^{2}+$ $\left\|\mathbf{u}^{+}\right\|_{0, \Omega_{+}}^{2}$. This result can be proved in two independent steps :

(i) It is worth noting that $\mathbf{u}^{-}$satisfies the following estimate

$$
\left\|\operatorname{curl} \mathbf{u}^{-}\right\|_{0, \Omega_{-}}+\left\|\operatorname{div} \mathbf{u}^{-}\right\|_{0, \Omega_{-}}+\left\|\mathbf{u}^{-} \times \mathbf{n}\right\|_{0, \Gamma} \gtrsim\left\|\mathbf{u}^{-}\right\|_{0, \Omega_{-}} .
$$

This is a direct consequence of the regularity result $\mathbf{u}^{-} \in \mathbf{H}^{\frac{1}{2}}\left(\Omega_{-}\right)$(see Remark 3.4) and the compact embedding of $\mathbf{H}^{\frac{1}{2}}\left(\Omega_{-}\right)$in $\mathbf{L}^{2}\left(\Omega_{-}\right)$.

(ii) The second step consists in proving the following estimate for $\mathbf{u}^{+}$

$$
\left\|\operatorname{curl} \mathbf{u}^{+}\right\|_{0, \Omega_{+}}+\left\|\operatorname{div} \mathbf{u}^{+}\right\|_{0, \Omega_{+}}+\left\|\mathbf{u}^{+} \times \mathbf{n}\right\|_{0, \Gamma} \gtrsim\left\|\mathbf{u}^{+}\right\|_{0, \Omega_{+}} .
$$

One can not apply the same regularity result mentioned above for $\mathbf{u}^{-}$to $\mathbf{u}^{+}$since the boundary of the domain $\Omega_{+}$is not connected. The proof of this estimate is based on a Helmholtz decomposition for $\mathbf{u}^{+}$(Section 3.5.1).

Finally combining estimates (3.16) and (3.17) we obtain the coerciveness result (3.14) which ends the proof of Lemma 3.6.

\subsubsection{Proof of Step (ii).}

Proof of (3.17). We recall that the domain $\Omega_{+}$is simply connected of class $\mathcal{C}^{1,1}$ and its boundary has two connected components $\partial \Omega_{+}=\Gamma \cup \partial \Omega$. One notes that $\mathbf{v}^{+}:=\operatorname{curl} \mathbf{u}^{+}$satisfies

$$
\operatorname{div} \mathbf{v}^{+}=0 \quad \text { in } \quad \Omega_{+}, \mathbf{v}^{+} \cdot \mathbf{n}=0 \quad \text { on } \quad \partial \Omega \quad \text { and } \quad\left\langle\mathbf{v}^{+} \cdot \mathbf{n}, 1\right\rangle_{\Gamma}=0 .
$$

Then relying to [9, Eq. (3.39)] and Theorem 2.12 in [2], we deduce that there exists a unique vector potential (with mixed boundary conditions) $\mathbf{w}^{+} \in \mathbf{H}^{1}\left(\Omega_{+}\right)$such that

$$
\begin{array}{r}
\operatorname{curl} \mathbf{w}^{+}=\operatorname{curl} \mathbf{u}^{+}, \quad \operatorname{div} \mathbf{w}^{+}=0 \quad \text { in } \Omega_{-}, \quad \mathbf{w}^{+} \cdot \mathbf{n}=0 \quad \text { on } \Gamma \\
\mathbf{w}^{+} \times \mathbf{n}=0 \quad \text { on } \partial \Omega, \quad \text { and }\left\langle\mathbf{w}^{+} \cdot \mathbf{n}, 1\right\rangle_{\partial \Omega}=0 .
\end{array}
$$

Moreover we have the estimate

$$
\left\|\mathbf{w}^{+}\right\|_{1, \Omega_{+}} \leqslant C\left\|\operatorname{curl} \mathbf{u}^{+}\right\|_{0, \Omega_{+}},
$$

where $C$ is independent of $\varepsilon$.

Since $\Omega_{+}$is simply connected and $\operatorname{curl} \mathbf{w}^{+}=\operatorname{curl} \mathbf{u}^{+}$in $\Omega_{+}$, we obtain that there exists $\varphi^{+} \in \mathrm{H}^{1}\left(\Omega_{+}\right)$(which is unique up to an additive constant) such that

$$
\mathbf{u}^{+}=\mathbf{w}^{+}+\nabla \varphi^{+} \quad \text { in } \quad \Omega_{+} .
$$


Thus $\varphi^{+}$is solution to the problem

$$
\Delta \varphi^{+}=\operatorname{div} \mathbf{u}^{+}, \quad \varphi^{+}=0 \quad \text { on } \partial \Omega \quad \text { and } \quad \int_{\Gamma} \partial_{\mathbf{n}} \varphi^{+} \mathrm{d} S=0 .
$$

Note that the gauge condition $\int_{\Gamma} \mathbf{u}^{+} \cdot \mathbf{n} \mathrm{d} S=0$ has been used here. According to Lemma 2.1 in [9], there exists a unique solution $\varphi^{+} \in \mathrm{H}_{0, \partial \Omega}^{1}\left(\Omega_{+}\right)=\left\{\varphi \in \mathrm{H}^{1}\left(\Omega_{+}\right) \mid \varphi=0 \quad\right.$ on $\left.\quad \partial \Omega\right\}$ to the problem (3.21) and we have a uniform estimate

$$
\left\|\varphi^{+}\right\|_{1, \Omega_{+}} \leqslant C\left(\left\|\operatorname{div} \mathbf{u}^{+}\right\|_{0, \Omega_{+}}+\left\|\left.\varphi^{+}\right|_{\Gamma}\right\|_{\mathrm{H}^{\frac{1}{2}(\Gamma) / \mathbb{C}}}\right) .
$$

It remains to bound $\left\|\left.\varphi^{+}\right|_{\Gamma}\right\|_{\mathrm{H}^{\frac{1}{2}}(\Gamma) / \mathbb{C}}$ since (3.19) and (3.20) implies that

$$
\left\|\mathbf{u}^{+}\right\|_{0, \Omega_{+}} \leqslant\left\|\mathbf{w}^{+}\right\|_{0, \Omega_{+}}+\left\|\nabla \varphi^{+}\right\|_{0, \Omega_{+}} \lesssim\left\|\operatorname{curl} \mathbf{u}^{+}\right\|_{0, \Omega_{+}}+\left\|\varphi^{+}\right\|_{1, \Omega_{+}} .
$$

Finally since we have the trace estimates

$$
\left\|\left.\varphi^{+}\right|_{\Gamma}\right\|_{\mathrm{H}^{\frac{1}{2}(\Gamma) / \mathbb{C}}} \leqslant\left\|\nabla \varphi^{+} \times \mathbf{n}\right\|_{-\frac{1}{2}, \Gamma} \leqslant\left\|\mathbf{u}^{+} \times \mathbf{n}\right\|_{-\frac{1}{2}, \Gamma}+\left\|\mathbf{w}^{+} \times \mathbf{n}\right\|_{-\frac{1}{2}, \Gamma}
$$

and

$$
\left\|\mathbf{u}^{+} \times \mathbf{n}\right\|_{-\frac{1}{2}, \Gamma} \leqslant\left\|\mathbf{u}^{+} \times \mathbf{n}\right\|_{0, \Gamma} \text { and }\left\|\mathbf{w}^{+} \times \mathbf{n}\right\|_{-\frac{1}{2}, \Gamma} \leqslant\left\|\mathbf{w}^{+}\right\|_{1, \Omega_{+}},
$$

we can combine the above estimates with (3.19) to infer the uniform estimate (3.17).

\section{VARIATIONAL FORMULATIONS FOR THE ELECTRIC FIELD. STABILITY AND CONVERGENCE RESULTS}

In this section we introduce variational formulations for the electric fields $\mathbf{E}^{\varepsilon}$ and $\mathbf{E}_{0}$, and we present elements of proofs for stability and convergence results.

\subsection{Strong form of equations.}

Equations for the electric field $\mathbf{E}^{\varepsilon}$. According to (2.1a)-(2.1b), the electric field $\mathbf{E}^{\varepsilon}$ solves the following problem for any $\varepsilon>0$ [9]

$$
\begin{aligned}
\operatorname{curl} \frac{1}{\mu_{ \pm}} \operatorname{curl} \mathbf{E}_{ \pm}^{\varepsilon} & =\mathrm{i} \omega \mathbf{j}^{ \pm} \quad \text { in } \Omega_{ \pm}^{\varepsilon}, \\
\operatorname{div} \mathbf{E}_{ \pm}^{\varepsilon} & =0 \quad \text { in } \Omega_{ \pm}^{\varepsilon}, \\
\operatorname{curl} \frac{1}{\mu_{o}} \operatorname{curl} \mathbf{E}_{o}^{\varepsilon}-\mathrm{i} \omega \sigma_{o}^{\varepsilon} \mathbf{E}_{o}^{\varepsilon} & =0 \quad \text { in } \Omega_{o}^{\varepsilon}, \\
\operatorname{div} \mathbf{E}_{o}^{\varepsilon} & =0 \text { in } \Omega_{o}^{\varepsilon}, \\
\mathbf{E}_{ \pm}^{\varepsilon} \times \mathbf{n} & =\mathbf{E}_{o}^{\varepsilon} \times \mathbf{n}, \quad \text { on } \Gamma_{ \pm}^{\varepsilon}, \\
\frac{1}{\mu_{ \pm}} \operatorname{curl} \mathbf{E}_{ \pm}^{\varepsilon} \times \mathbf{n} & =\frac{1}{\mu_{o}} \operatorname{curl} \mathbf{E}_{o}^{\varepsilon} \times \mathbf{n}, \quad \text { on } \Gamma_{ \pm}^{\varepsilon}, \\
\int_{\Gamma_{+}^{\varepsilon}} \mathbf{E}_{+}^{\varepsilon} \cdot \mathbf{n} \mathrm{d} S & =0, \\
\mathbf{E}_{+}^{\varepsilon} \times \mathbf{n} & =0 \quad \text { on } \partial \Omega .
\end{aligned}
$$


Equations for the electric field $\mathbf{E}_{0}$. The electric field $\mathbf{E}_{0}^{-}$solves the following problem

$$
\begin{array}{rc}
\operatorname{curl} \frac{1}{\mu_{-}} \operatorname{curl} \mathbf{E}_{0}^{-}=\mathrm{i} \omega \mathbf{j}^{-} & \text {in } \quad \Omega_{-}, \\
\operatorname{div} \mathbf{E}_{0}^{-}=0 \quad \text { in } \quad \Omega_{-}, \\
\mathbf{E}_{0}^{-} \times \mathbf{n}=0 \quad \text { on } \Gamma,
\end{array}
$$

and $\mathbf{E}_{0}^{+}$solves the following problem

$$
\begin{array}{rlrl}
\operatorname{curl} \frac{1}{\mu_{+}} \operatorname{curl} \mathbf{E}_{0}^{+} & =\mathrm{i} \omega \mathbf{j}^{+} \quad \text { in } \quad \Omega_{+}, \\
\operatorname{div} \mathbf{E}_{0}^{+} & =0 \quad \text { in } \quad \Omega_{+}, \\
\int_{\Gamma} \mathbf{E}_{0}^{+} \cdot \mathbf{n} \mathrm{d} S & =0, & & \\
\mathbf{E}_{0}^{+} \times \mathbf{n} & =0 \quad & & \text { on } \Gamma \cup \partial \Omega .
\end{array}
$$

4.2. Variational framework. The variational space for $\mathbf{E}^{\varepsilon}$ is the Hilbert space

$$
\mathbf{Y}_{\varepsilon}=\left\{\mathbf{u} \in \mathbf{H}_{0}(\operatorname{curl}, \Omega): \operatorname{div} \mathbf{u}_{ \pm} \in \mathrm{L}^{2}\left(\Omega_{ \pm}^{\varepsilon}\right), \operatorname{div} \mathbf{u}_{o} \in \mathrm{L}^{2}\left(\Omega_{o}^{\varepsilon}\right), \int_{\Gamma_{ \pm}^{\varepsilon}} \mathbf{u}_{ \pm} \cdot \mathbf{n} \mathrm{d} S=0\right\}
$$

equipped with the norm

$$
\|\mathbf{u}\|_{\mathbf{Y}_{\varepsilon}}^{2}=\|\mathbf{u}\|_{0, \Omega}^{2}+\|\operatorname{curl} \mathbf{u}\|_{0, \Omega}^{2}+\left\|\operatorname{div} \mathbf{u}_{+}\right\|_{0, \Omega_{+}^{\varepsilon}}^{2}+\left\|\operatorname{div} \mathbf{u}_{-}\right\|_{0, \Omega_{-}^{\varepsilon}}^{2}+\left\|\operatorname{div} \mathbf{u}_{o}\right\|_{0, \Omega_{o}^{\varepsilon}}^{2} .
$$

The variational spaces for $\mathbf{E}_{0}^{+}$and $\mathbf{E}_{0}^{-}$are

$$
\mathbf{Y}_{0}\left(\Omega_{+}\right)=\left\{\mathbf{u} \in \mathbf{H}_{0}\left(\operatorname{curl}, \Omega_{+}\right): \operatorname{div} \mathbf{u} \in \mathrm{L}^{2}\left(\Omega_{+}\right), \int_{\Gamma} \mathbf{u} \cdot \mathbf{n} \mathrm{d} S=0\right\}
$$

and

$$
\mathbf{Y}_{0}\left(\Omega_{-}\right)=\left\{\mathbf{u} \in \mathbf{H}_{0}\left(\operatorname{curl}, \Omega_{-}\right): \operatorname{div} \mathbf{u} \in \mathrm{L}^{2}\left(\Omega_{-}\right), \int_{\Gamma} \mathbf{u} \cdot \mathbf{n} \mathrm{d} S=0\right\},
$$

respectively. The spaces $\mathbf{Y}_{0}\left(\Omega_{+}\right)$and $\mathbf{Y}_{0}\left(\Omega_{-}\right)$are equipped with the norms $\|\cdot\|_{\mathbf{Y}_{0}\left(\Omega_{+}\right)}$and $\|\cdot\|_{\mathbf{Y}_{0}\left(\Omega_{-}\right)}$, respectively

$$
\|\mathbf{u}\|_{\mathbf{Y}_{0}\left(\Omega_{ \pm}\right)}^{2}=\|\mathbf{u}\|_{0, \Omega_{ \pm}}^{2}+\|\operatorname{curl} \mathbf{u}\|_{0, \Omega_{ \pm}}^{2}+\|\operatorname{div} \mathbf{u}\|_{0, \Omega_{ \pm}}^{2} .
$$

\subsection{Variational problems.}

Variational problem for the electric field $\mathbf{E}^{\varepsilon}$. For all $\varepsilon>0$ we consider the variational problem :

Find $\mathbf{E} \in \mathbf{Y}_{\varepsilon}$ such that for all $\mathbf{v} \in \mathbf{Y}_{\varepsilon}$,

$$
a_{R}^{\varepsilon}(\mathbf{E}, \mathbf{v})=\mathrm{i} \omega \int_{\Omega_{-}^{\varepsilon} \cup \Omega_{+}^{\varepsilon}} \mathbf{j} \cdot \overline{\mathbf{v}} \mathrm{d} \mathbf{x} .
$$

Here the sesquilinear form (in its regularized version) $a_{R}^{\varepsilon}$ is defined as

$$
a_{R}^{\varepsilon}(\mathbf{u}, \mathbf{v})=\int_{\Omega} \frac{1}{\underline{\mu}^{\varepsilon}} \operatorname{curl} \mathbf{u} \cdot \operatorname{curl} \overline{\mathbf{v}} \mathrm{d} \mathbf{x}+\int_{\Omega_{-}^{\varepsilon} \cup \Omega_{o}^{\varepsilon} \cup \Omega_{+}^{\varepsilon}} \operatorname{div} \mathbf{u} \operatorname{div} \overline{\mathbf{v}} \mathrm{d} \mathbf{x}-\mathrm{i} \omega \int_{\Omega_{o}^{\varepsilon}} \sigma_{o}^{\varepsilon} \mathbf{u} \cdot \overline{\mathbf{v}} \mathrm{d} \mathbf{x} .
$$


Lemma 4.1. Let the positive constants $\mu_{ \pm}, \mu_{o}, \tilde{\sigma}$ and $\omega$ be fixed. Then there exists $\varepsilon_{0}>0$ such that for all $\varepsilon \in\left(0, \varepsilon_{0}\right), a_{R}^{\varepsilon}$ is strongly coercive on $\mathbf{Y}_{\varepsilon}:$ there exist $\alpha \in \mathbb{C}$ and $c_{0}>0$ such that for all $\varepsilon \in\left(0, \varepsilon_{0}\right)$ and for all $\mathbf{E} \in \mathbf{Y}_{\varepsilon}$

$$
\operatorname{Re}\left(\alpha a_{R}^{\varepsilon}(\mathbf{E}, \mathbf{E})\right) \geqslant c_{0}\|\mathbf{E}\|_{\mathbf{Y}_{\varepsilon}}^{2} .
$$

Proof. Let us fix $\alpha=\mathrm{e}^{\mathrm{i} \pi / 4}$. Since

$\operatorname{Re}\left(\alpha a_{R}^{\varepsilon}(\mathbf{E}, \mathbf{E})\right) \gtrsim\|\operatorname{curl} \mathbf{E}\|_{0, \Omega}^{2}+\left\|\operatorname{div} \mathbf{E}_{+}\right\|_{0, \Omega_{+}^{\varepsilon}}^{2}+\left\|\operatorname{div} \mathbf{E}_{-}\right\|_{0, \Omega_{-}^{\varepsilon}}^{2}+\left\|\operatorname{div} \mathbf{E}_{o}\right\|_{0, \Omega_{o}^{\varepsilon}}^{2}+\varepsilon^{-2}\|\mathbf{E}\|_{0, \Omega_{o}^{\varepsilon}}^{2}$, then there exists $\varepsilon_{0}>0$ such that for all $\varepsilon \in\left(0, \varepsilon_{0}\right)$ and for all $\mathbf{E} \in \mathbf{Y}_{\varepsilon}$

$$
\operatorname{Re}\left(\alpha a_{R}^{\varepsilon}(\mathbf{E}, \mathbf{E})\right) \gtrsim\|\operatorname{curl} \mathbf{E}\|_{0, \Omega}^{2}+\left\|\operatorname{div} \mathbf{E}_{+}\right\|_{0, \Omega_{+}^{\varepsilon}}^{2}+\left\|\operatorname{div} \mathbf{E}_{-}\right\|_{0, \Omega_{-}^{\varepsilon}}^{2}+\left\|\operatorname{div} \mathbf{E}_{o}\right\|_{0, \Omega_{o}^{\varepsilon}}^{2}+\|\mathbf{E}\|_{0, \Omega_{o}^{\varepsilon}}^{2} .
$$

Then it is possible to prove that the right hand side is an upper bound for $\|\mathbf{E}\|_{0, \Omega_{-}^{\varepsilon}}^{2}+\|\mathbf{E}\|_{0, \Omega_{+}^{\varepsilon}}^{2}$. The proof is worked out in details in [9, Lemma 2.2] (with $\delta_{0}=0$ ) in a slightly different configuration.

Variational problems for $\mathbf{E}_{0}^{ \pm}$. We consider the variational problems :

(i) Find $\mathbf{E}_{0}^{-} \in \mathbf{Y}_{0}\left(\Omega_{-}\right)$such that for all $\mathbf{v} \in \mathbf{Y}_{0}\left(\Omega_{-}\right)$,

$$
a_{-}^{0}\left(\mathbf{E}_{0}^{-}, \mathbf{v}\right)=\mathrm{i} \omega \int_{\Omega_{-}} \mathbf{j}^{-} \cdot \overline{\mathbf{v}} \mathrm{d} \mathbf{x}
$$

(ii) Find $\mathbf{E}_{0}^{+} \in \mathbf{Y}_{0}\left(\Omega_{+}\right)$such that for all $\mathbf{v} \in \mathbf{Y}_{0}\left(\Omega_{+}\right)$,

$$
a_{+}^{0}\left(\mathbf{E}_{0}^{+}, \mathbf{v}\right)=\mathrm{i} \omega \int_{\Omega_{+}} \mathbf{j}^{+} \cdot \overline{\mathbf{v}} \mathrm{d} \mathbf{x} .
$$

Here the sesquilinear forms (regularized versions) $a_{ \pm}^{0}$ are defined as

$$
a_{ \pm}^{0}(\mathbf{u}, \mathbf{v})=\int_{\Omega_{ \pm}} \frac{1}{\mu_{ \pm}} \operatorname{curl} \mathbf{u} \cdot \operatorname{curl} \overline{\mathbf{v}} \mathrm{d} \mathbf{x}+\int_{\Omega_{ \pm}} \operatorname{div} \mathbf{u} \operatorname{div} \overline{\mathbf{v}} \mathrm{d} \mathbf{x}
$$

We have the following well-posedness and elliptic regularity results.

Proposition 4.2. Let $\mathbf{j} \in \mathbf{L}^{2}(\Omega)$ such that $\operatorname{div} \mathbf{j}^{ \pm}=0$ in $\Omega_{ \pm}$. Then there exists a unique solution $\mathbf{E}_{0}^{-} \in \mathbf{Y}_{0}\left(\Omega_{-}\right)$to problem (4.6) and there exists a unique solution $\mathbf{E}_{0}^{+} \in \mathbf{Y}_{0}\left(\Omega_{+}\right)$to problem (4.7). The solution $\mathbf{E}_{0}^{ \pm}$satisfies all equations in (4.2)-(4.3). Furthermore, if $\mathbf{j}^{ \pm} \in \mathbf{H}^{s}\left(\Omega_{ \pm}\right), s \geqslant 0$, then we have

$$
\mathbf{E}_{0}^{-} \in \mathbf{H}^{s+2}\left(\Omega_{-}\right) \quad \text { and } \quad \mathbf{E}_{0}^{+} \in \mathbf{H}^{s+2}\left(\Omega_{+}\right) .
$$

Remark 4.3. Since the surface $\partial \Omega_{-}$is connected, the semi-norm

$$
\mathbf{u} \longmapsto\|\operatorname{curl} \mathbf{u}\|_{0, \Omega_{-}}+\|\operatorname{div} \mathbf{u}\|_{0, \Omega_{-}},
$$

on the space $\mathbf{X}_{\mathbf{N}}\left(\Omega_{-}\right)=\mathbf{H}\left(\operatorname{div}, \Omega_{-}\right) \cap \mathbf{H}_{0}\left(\operatorname{curl}, \Omega_{-}\right)$is equivalent to the norm $\|\cdot\|_{\mathbf{X}\left(\Omega_{-}\right)}$where $\mathbf{X}\left(\Omega_{-}\right)=\mathbf{H}\left(\operatorname{div}, \Omega_{-}\right) \cap \mathbf{H}\left(\operatorname{curl}, \Omega_{-}\right)$(See e.g. [2, Corollary 3.19]). Then the well-posedness result for $\mathbf{E}_{0}^{-}$in $\mathbf{Y}_{0}\left(\Omega_{-}\right)$can be obtained as an application of the Lax-Milgram Lemma.

As a consequence of Lemma 4.1, we infer the following theorem 
Theorem 4.4. Let $\mathbf{j} \in \mathbf{L}^{2}(\Omega)$ such that $\operatorname{div} \mathbf{j}=0$ in $\Omega_{ \pm}^{\varepsilon}, \mathbf{j}=0$ in $\Omega_{o}^{\varepsilon}$ and $\mathbf{j} \cdot \mathbf{n}=0$ on $\Gamma_{ \pm}^{\varepsilon}$. Let the positive constants $\mu_{ \pm}, \mu_{o}, \tilde{\sigma}$ and $\omega$ be fixed. Then there exists $\varepsilon_{0}>0$ such that for all $\varepsilon \in\left(0, \varepsilon_{0}\right)$

(i) There exists a unique solution $\mathbf{E}^{\varepsilon} \in \mathbf{Y}_{\varepsilon}$ to problem (4.4).

(ii) The solution $\mathbf{E}^{\varepsilon}$ satisfies all equations in (4.1).

(iii) Uniform estimates in $\mathbf{Y}_{\varepsilon}$ : there exists $C>0$ such that for all $\varepsilon \in\left(0, \varepsilon_{0}\right)$

$$
\left\|\mathbf{E}^{\varepsilon}\right\|_{\mathbf{Y}_{\varepsilon}} \leqslant C\|\mathbf{j}\|_{0, \Omega} .
$$

(iv) As $\varepsilon \rightarrow 0, \mathbf{E}^{\varepsilon} \rightarrow \mathbf{E}_{0}$ (at least in $\mathbf{L}^{2}\left(\Omega_{o}^{\varepsilon}\right)$ ) and we have the following estimates : there exists $C>0$ such that for any small parameter $\varepsilon \in\left(0, \varepsilon_{0}\right)$

$$
\left\|\mathbf{E}^{\varepsilon}-\mathbf{E}_{0}\right\|_{0, \Omega_{o}^{\varepsilon}}+\varepsilon\left\|\operatorname{curl}\left(\mathbf{E}^{\varepsilon}-\mathbf{E}_{0}\right)\right\|_{0, \Omega} \leqslant C \sqrt{\varepsilon} .
$$

Proof. (i) Accordingly estimate (4.5), a straightforward application of the Lax-Milgram lemma leads to existence and uniqueness to the solution $\mathbf{E}^{\varepsilon}$ to the variational problem (4.4).

(ii) The proof is worked out in details in [9, Theorem 2.3] in a slightly different configuration.

(iii) Using estimates (4.5), estimates (4.8) are obvious.

(iv) According to Prop. 4.2, $\mathbf{E}_{0}^{ \pm}$and curl $\mathbf{E}_{0}^{ \pm}$belong to $\mathbf{H}^{2}\left(\Omega_{ \pm}\right)$. Hence $\mathbf{E}_{0}^{ \pm}$and curl $\mathbf{E}_{0}^{ \pm}$belong to $\mathbf{L}^{\infty}\left(\Omega_{ \pm}\right)$. We denote by $\mathbf{U}=\mathbf{E}^{\varepsilon}-\mathbf{E}_{0}$.

According to (4.1)-(4.2), and since $\operatorname{curl} \frac{1}{\mu_{o}} \operatorname{curl} \mathbf{E}_{0}^{ \pm}=\mathrm{i} \omega \mathbf{j}^{ \pm}=0$ in $\Omega_{o}^{\varepsilon}$, we deduce that $\mathbf{U}$ solves the following equations for any $\varepsilon>0$

$$
\begin{aligned}
\operatorname{curl} \frac{1}{\mu_{ \pm}} \operatorname{curl} \mathbf{U}_{ \pm} & =0 \quad \text { in } \Omega_{ \pm}^{\varepsilon}, \\
\operatorname{div} \mathbf{U}_{ \pm} & =0 \quad \text { in } \Omega_{ \pm}^{\varepsilon}, \\
\operatorname{curl} \frac{1}{\mu_{o}} \operatorname{curl} \mathbf{U}_{o}-\mathrm{i} \omega \sigma_{o}^{\varepsilon} \mathbf{U}_{o} & =\mathrm{i} \omega \sigma_{o}^{\varepsilon} \mathbf{E}_{0}^{-} \quad \text { in } \Omega_{o}^{\varepsilon} \cap \Omega_{-}, \\
\operatorname{curl} \frac{1}{\mu_{o}} \operatorname{curl} \mathbf{U}_{o}-\mathrm{i} \omega \sigma_{o}^{\varepsilon} \mathbf{U}_{o} & =\mathrm{i} \omega \sigma_{o}^{\varepsilon} \mathbf{E}_{0}^{+} \quad \text { in } \quad \Omega_{o}^{\varepsilon} \cap \Omega_{+}, \\
\operatorname{div} \mathbf{U}_{o} & =0 \quad \text { in } \Omega_{o}^{\varepsilon} \cap\left(\Omega_{-} \cup \Omega_{+}\right), \\
{[\mathbf{U} \times \mathbf{n}] } & =0, \quad \text { on } \Gamma_{ \pm}^{\varepsilon} \cup \Gamma, \\
{\left[\frac{1}{\mu} \operatorname{curl} \mathbf{U} \times \mathbf{n}\right] } & =0, \quad \text { on } \Gamma_{ \pm}^{\varepsilon}, \\
\left.\frac{1}{\mu} \operatorname{curl} \mathbf{U} \times \mathbf{n}\right] & =-\left[\frac{1}{\mu} \operatorname{curl} \mathbf{E}_{0} \times \mathbf{n}\right], \text { on } \Gamma, \\
\mathbf{U}+\times \mathbf{n} & =0 \quad \text { on } \partial \Omega .
\end{aligned}
$$

One notes that curl $\mathbf{U}$ belongs to $\mathbf{L}^{2}(\Omega)$. Multiplying the equations (4.10a)-(4.10c)-(4.10d) by $\overline{\mathbf{U}}$ and integrating by parts we obtain the following identity

$$
\begin{aligned}
\int_{\Omega} \frac{1}{\mu^{\varepsilon}}|\operatorname{curl} \mathbf{U}|^{2} \mathrm{~d} \mathbf{x}-\mathrm{i} \omega \varepsilon^{-2} \tilde{\sigma} \int_{\Omega_{o}^{\varepsilon}}\left|\mathbf{U}_{o}\right|^{2} \mathrm{~d} \mathbf{x}= & \\
& \left.\quad \mathrm{i} \omega \varepsilon^{-2} \tilde{\sigma} \int_{\Omega_{o}^{\varepsilon}} \mathbf{E}_{0} \cdot \overline{\mathbf{U}_{o}} \mathrm{~d} \mathbf{x}+\mathrm{i} \omega\left\langle\left[\mathbf{H}_{0} \times \mathbf{n}\right]_{\Gamma}, \overline{\left(\mathbf{E}_{o}^{\varepsilon}\right.}\right)_{\mathbf{T}}\right\rangle_{\Gamma},
\end{aligned}
$$

since $\left[\frac{1}{\mu} \operatorname{curl} \mathbf{E}_{0} \times \mathbf{n}\right]=-\mathrm{i} \omega\left[\mathbf{H}_{0} \times \mathbf{n}\right]$ and $\mathbf{U}_{\mathbf{T}}=\left(\mathbf{E}_{o}^{\varepsilon}\right)_{\mathbf{T}}$ on $\Gamma$. 
One notes that $\left[\mathbf{H}_{0} \times \mathbf{n}\right]_{\Gamma}$ belongs to $\mathbf{H}^{\frac{1}{2}}(\Gamma)$ and according to (4.8) $\left(\mathbf{E}_{o}^{\varepsilon}\right)_{\mathbf{T}}$ is bounded in $\mathbf{H}^{-\frac{1}{2}}(\Gamma)$. Then taking the imaginary part and the real part of the identity (4.11) and absorbing the right-hand sides, we infer the following estimates

$$
\|\mathbf{U}\|_{0, \Omega_{o}^{\varepsilon}}+\varepsilon\|\operatorname{curl} \mathbf{U}\|_{0, \Omega} \leqslant C \sqrt{\varepsilon},
$$

since

$$
\left\|\mathbf{E}_{0}\right\|_{0, \Omega_{o}^{\varepsilon}} \lesssim \sqrt{\varepsilon}
$$

That ends the proof of estimates (4.9).

\section{A MULTISCALE EXPANSION FOR THE MAGNETIC FIELD}

In the guidelines on the derivation of the equivalent transmission conditions in Sect. 2.4 we have already argued that this derivation is based on an asymptotic expansion for the magnetic field $\mathbf{H}^{\varepsilon}$ (2.2) inside and outside the sheet. More precisely, we search $\mathbf{H}^{\varepsilon}$ as the asymptotic expansion (2.3), which is

$$
\begin{array}{ll}
\mathbf{H}^{\varepsilon}(\mathbf{x}) \approx \mathbf{H}_{0}(\mathbf{x})+\varepsilon \mathbf{H}_{1}(\mathbf{x})+\varepsilon^{2} \mathbf{H}_{2}(\mathbf{x})+\cdots \quad & \text { for a.e. } \quad \mathbf{x} \in \Omega_{-}^{\varepsilon} \cup \Omega_{+}^{\varepsilon}, \\
\mathbf{H}^{\varepsilon}(\mathbf{x}) \approx \underline{\mathfrak{H}}_{0}\left(y_{\alpha}, \frac{h}{\varepsilon}\right)+\varepsilon \underline{\mathfrak{Y}}_{1}\left(y_{\alpha}, \frac{h}{\varepsilon}\right)+\cdots & \text { for a.e. } \quad \mathbf{x} \in \Omega_{o}^{\varepsilon} .
\end{array}
$$

In this section, we will derive the terms of this asymptotic expansion step by step up to order 2 as well as their governing equations, having in mind that the magnetic field $\mathbf{H}^{\varepsilon}$ satisfies the following transmission problem

$$
\begin{aligned}
\operatorname{curl} \mathbf{H}_{ \pm}^{\varepsilon} & =\mathbf{j}_{ \pm} & & \text {in } \Omega_{ \pm}^{\varepsilon}, \\
\operatorname{div} \mathbf{H}_{ \pm}^{\varepsilon} & =0 & & \text { in } \Omega_{ \pm}^{\varepsilon}, \\
\operatorname{curlcurl} \mathbf{H}_{o}^{\varepsilon}-\left(\kappa_{o}^{\varepsilon}\right)^{2} \mathbf{H}_{o}^{\varepsilon} & =0 & & \text { in } \Omega_{o}^{\varepsilon}, \\
\mathbf{H}_{ \pm}^{\varepsilon} \times \mathbf{n} & =\mathbf{H}_{o}^{\varepsilon} \times \mathbf{n}, & & \text { on } \Gamma_{ \pm}^{\varepsilon}, \\
\mu_{ \pm} \mathbf{H}_{ \pm}^{\varepsilon} \cdot \mathbf{n} & =\mu_{o} \mathbf{H}_{o}^{\varepsilon} \cdot \mathbf{n}, & & \text { on } \Gamma_{ \pm}^{\varepsilon} \\
\mathbf{H}^{\varepsilon} \cdot \mathbf{n} & =0 & & \text { on } \partial \Omega .
\end{aligned}
$$

In (5.1c) we denote by $\kappa_{o}^{\varepsilon}$ the complex wave number given by

$$
\kappa_{o}^{\varepsilon}=\varepsilon^{-1} \sqrt{\omega \mu_{o} \tilde{\sigma}} \mathrm{e}^{\mathrm{i} \pi / 4} .
$$

This derivation is order by order and for each order $n$ it is in four steps:

(i) Writing the operator curl curl in the domain $\Omega_{o}^{\varepsilon}$ in local coordinates $\left(y_{\alpha}, h\right)[7]$ and performing the change of variable $Y_{3}=\varepsilon^{-1} h$, i.e., rewriting it in in $\left(y_{\alpha}, Y_{3}\right)$-coordinates, leads to an algebraic equation fixing the normal component $\mathfrak{h}_{n}$ of $\underline{\mathfrak{Y}}_{n}$ and a differential equation for the two tangential components $\mathfrak{H}_{n}$, which are not completely defined yet.

(ii) We expand the far field term $\mathbf{H}_{n}$ at the two surfaces $\Gamma_{ \pm}^{\varepsilon}$ of the conductor around its mid-surface $\Gamma$ using the Taylor expansion.

(iii) Using the the transmission conditions (5.1e) on $\Gamma_{ \pm}^{\varepsilon}$ and the Taylor expansion of $\mathbf{H}_{n}$ we obtain boundary conditions for $\mathbf{H}_{n}^{ \pm}$on $\Gamma$, which depend on the first terms $\mathbf{H}_{k}, k=$ $0, \ldots, n-1$ on $\Gamma$. 
(iv) Using the transmission conditions (5.1d) on $\Gamma_{ \pm}^{\varepsilon}$ together with the Taylor expansion of $\mathbf{H}_{n}$ we obtain boundary conditions for the tangential components $\mathfrak{H}_{n}$ inside the sheet. They can now be explicitly defined as a function of the terms $\mathbf{H}_{k}, k=0, \ldots, n$.

We expand the "magnetic" operator inside the thin layer $\Omega_{o}^{\varepsilon}$ in powers of $\varepsilon$, in Section 5.1. We deduce in Section 5.2 the equations satisfied by the magnetic profiles $\underline{\mathfrak{Y}}_{n}$ and the far field terms $\mathbf{H}_{n}^{ \pm}$. We derive explicitly the first terms in Section 5.3.

5.1. Expansion of differential operators inside the conductor. Due to the small thickness of the conductor the derivatives in normal and the tangential directions scale differently in $\varepsilon$. Hence, it is convenient to use a local normal coordinate system in $\Omega_{o}^{\varepsilon}$, see e.g., [7, App. A.1]. For this coordinate system we call $D_{\alpha}$ the covariant derivative on the mean surface $\Gamma$ and $\partial_{3}^{h}$ is the partial derivative with respect to the normal coordinate $y_{3}=h$. Let furthermore $a_{\alpha \beta}(h)$ be the metric tensor of the manifold $\Gamma_{h}$, which is the surface contained in $\Omega_{o}^{\varepsilon}$ at a distance $h$ of $\Gamma$. The metric tensor in such a coordinate system writes [7, App. A.1, Eq. (A.7)]

$$
a_{\alpha \beta}(h)=a_{\alpha \beta}-2 b_{\alpha \beta} h+b_{\alpha}^{\gamma} b_{\gamma \beta} h^{2},
$$

and its inverse expands in power series of $h$

$$
a^{\alpha \beta}(h)=a^{\alpha \beta}+2 b^{\alpha \beta} h+\mathcal{O}\left(h^{2}\right) .
$$

Subsequently, we use a property of the covariant derivative, that it acts on scalar functions $\mathfrak{h}$ like the partial derivative: $D_{\alpha} \mathfrak{h}=\partial_{\alpha} \mathfrak{h}$.

We denote by $\mathbf{L}\left(y_{\alpha}, h ; D_{\alpha}, \partial_{3}^{h}\right)$ the second order Maxwell operator

$$
\text { curl curl }-\left(\kappa_{o}^{\varepsilon}\right)^{2} \mathbb{I}
$$

in $\Omega_{o}^{\varepsilon}$ in the normal coordinate system. The operator $\mathbf{L}$ expand in power series of $h$ with intrinsic coefficients with respect to $\Gamma$, see [7, App. A, §A.4].

Now, we scale the normal coordinate $Y_{3}=\varepsilon^{-1} h$ to obtain a coordinate, this is $Y_{3}$, which does not change with $\varepsilon$. We use from now on the same symbol $\mathfrak{H}$ for three-dimensional one-form field in these scaled coordinates and call $\mathbf{L}[\varepsilon]$ the three-dimensional harmonic Maxwell operators in $\Omega_{o}^{\varepsilon}$. This operator expands in powers of $\varepsilon$

$$
\mathbf{L}[\varepsilon]=\varepsilon^{-2} \sum_{n=0}^{\infty} \varepsilon^{n} \mathbf{L}^{n},
$$

whose coefficients are intrinsic operators on $\Gamma$, which are completely determined by the shape of $\Gamma$ and the material parameters of the conducting sheet. We denote by $L_{\alpha}^{n}$ the surface components of $\mathbf{L}^{n}$. With the summation convention of repeated two dimensional indices (represented by greek letters), there holds [7, App. A.1, Eq. (A.10)]

$$
L_{\alpha}^{0}(\underline{\mathfrak{H}})=-\partial_{3}^{2} \mathfrak{H}_{\alpha}+\gamma^{2} \mathfrak{H}_{\alpha} \text { and } L_{\alpha}^{1}(\underline{\mathfrak{H}})=-2 b_{\alpha}^{\beta} \partial_{3} \mathfrak{H}_{\beta}+\partial_{3} D_{\alpha} \mathfrak{h}+2 H \partial_{3} \mathfrak{H}_{\alpha},
$$

(we recall that $\gamma$ is defined in (3.5), so that $\left(\kappa_{o}^{\varepsilon}\right)^{2}=-\varepsilon^{-2} \gamma^{2}$ ). Here, $\partial_{3}$ is the partial derivative with respect to $Y_{3}$ and $H=\frac{1}{2} b_{\beta}^{\beta}$ is the mean curvature of the surface $\Gamma$. We denote by $L_{3}^{n}$ the transverse components of $\mathbf{L}^{n}$. There holds [7, App. A.1, Eq. (A.12)]

$$
L_{3}^{0}(\underline{\mathfrak{Y}})=\gamma^{2} \mathfrak{h} \quad \text { and } \quad L_{3}^{1}(\underline{\mathfrak{H}})=\gamma_{\alpha}^{\alpha}\left(\partial_{3} \underline{\mathfrak{H}}\right)+2 H \partial_{3} \mathfrak{h},
$$

where $\gamma_{\alpha \beta}(\underline{\mathfrak{H}})=\frac{1}{2}\left(D_{\alpha} \mathfrak{H}_{\beta}+D_{\beta} \mathfrak{H}_{\alpha}\right)-b_{\alpha \beta} \mathfrak{h}$ is the change of metric tensor and $\gamma_{\alpha}^{\alpha}=a^{\alpha \beta} \gamma_{\alpha \beta}$. 
5.2. Equations for the coefficients of the magnetic field. Writing the partial differential equation (5.1c) in the thin conductor $\Omega_{o}^{\varepsilon}$ in the scaled local coordinate system we find that the profiles $\underline{\mathfrak{T}}_{j}$ satisfy the following system (with $I=\left(-\frac{1}{2}, \frac{1}{2}\right)$ )

$$
\mathbf{L}[\varepsilon] \sum_{j \geqslant 0} \varepsilon^{j} \underline{\mathfrak{T}}_{j}\left(y_{\alpha}, Y_{3}\right)=0, \quad \text { in } \Gamma \times I .
$$

It is not very convenient that the terms $\mathbf{H}_{j}^{ \pm}$of the magnetic field (see (2)) (which are involved on the left hand side of (5.1d)-(5.1e)) are evaluated on $\Gamma_{ \pm}^{\varepsilon}$ which moves with $\varepsilon$. However, as the expansion (2) of $\mathbf{H}^{\varepsilon}$ is assumed to be valid for any small $\varepsilon>0$, the terms $\mathbf{H}_{j}^{ \pm}$are defined in $\Omega_{ \pm}^{\varepsilon}$ for any $\varepsilon>0$, and, hence, in $\Omega_{ \pm}$. As we have assumed that the thin conductor, and so its mid-surface $\Gamma$, are smooth, that $\mu_{ \pm}$are constants, and that the current $\mathbf{j}$ is zero close to $\Gamma$ it makes sense to assume that the vector fields $\mathbf{H}_{j}^{ \pm}$are regular in a neighbourhood of $\Gamma$. This can be justified using the regularity theory, see e.g. [23, Chap. 4]. Hence, we can use the Taylor expansion and infer for $n \in \mathbb{N}$ that

$$
\mathbf{H}_{n}^{ \pm} \times\left.\mathbf{n}\right|_{h= \pm \frac{\varepsilon}{2}}=\quad \mathbf{H}_{n} \times\left.\mathbf{n}\right|_{0^{ \pm}} \pm \frac{\varepsilon}{2} \partial_{h} \mathbf{H}_{n} \times\left.\mathbf{n}\right|_{0^{ \pm}}+\cdots,
$$

and

$$
\left.\mathbf{H}_{n}^{ \pm} \cdot \mathbf{n}\right|_{h= \pm \frac{\varepsilon}{2}}=\left.\quad \mathbf{H}_{n} \cdot \mathbf{n}\right|_{0^{ \pm}} \pm\left.\frac{\varepsilon}{2} \partial_{h} \mathbf{H}_{n} \cdot \mathbf{n}\right|_{0^{ \pm}}+\cdots,
$$

where $\left.\cdot\right|_{0^{ \pm}}$means the limit for positive or negative $h \rightarrow 0$, respectively. Furthermore, it is convenient to define $\mathbf{H}_{n}$ for $n \in \mathbb{N}$ by $\mathbf{H}_{n}=\mathbf{H}_{n}^{+}$in $\Omega_{+}$, and $\mathbf{H}_{n}=\mathbf{H}_{n}^{-}$in $\Omega_{-}$.

Using the expression of the operator $\mathbf{L}^{0}$, and expanding $\mathbf{H}^{\varepsilon}$ in $\Omega_{ \pm}^{\varepsilon}$, we deduce that, according to the system (5.1) and using (5.5) and (5.6), the profiles $\underline{\mathfrak{H}}_{n}=\left(\mathfrak{H}_{n}, \mathfrak{h}_{n}\right)$ and the terms $\mathbf{H}_{n}$ have to satisfy, for all $n \geq 0$

$$
\begin{array}{rlrl}
L_{3}^{0}\left(\underline{\mathfrak{H}}_{n}\right)=\gamma^{2} \mathfrak{h}_{n} & =-\sum_{j=1}^{n} L_{3}^{j}\left(\underline{\mathfrak{Y}}_{n-j}\right) & \text { in } \quad \Gamma \times I, \\
\operatorname{curl} \mathbf{H}_{n}^{ \pm} & =\delta_{n}^{0} \mathbf{j}_{ \pm} & \text {in } \quad \Omega_{ \pm}, \\
\operatorname{div} \mathbf{H}_{n}^{ \pm} & =0 & \text { in } \quad \Omega_{ \pm}, \\
\left.\mathbf{H}_{n}^{ \pm} \cdot \mathbf{n}\right|_{0^{ \pm}} & =\left.\frac{\mu_{o}}{\mu_{ \pm}} \mathfrak{h}_{n}\right|_{ \pm \frac{1}{2}}-\left.\sum_{j=1}^{n} \frac{1}{( \pm 2)^{j}} \partial_{h}^{j} \mathbf{H}_{n-j}^{ \pm} \cdot \mathbf{n}\right|_{0^{ \pm}} \\
\mathbf{H}_{n}^{+} \cdot \mathbf{n} & =0 & \text { on } \quad \Gamma, \\
L_{\alpha}^{0}\left(\underline{\mathfrak{H}}_{n}\right)=-\partial_{3}^{2} \mathfrak{H}_{n, \alpha}+\gamma^{2} \mathfrak{H}_{n, \alpha} & =-\sum_{j=1}^{n} L_{\alpha}^{j}\left(\underline{\mathfrak{H}}_{n-j}\right) & \text { on } \quad \partial \Omega, \\
\left.\mathfrak{H}_{n}\right|_{ \pm \frac{1}{2}} & =\mathbf{n} \times \mathbf{h}_{n}^{ \pm} \times\left.\mathbf{n}\right|_{0^{ \pm}}+\sum_{j=1}^{n} \frac{1}{( \pm 2)^{j}} \partial_{h}^{j} \mathbf{n} \times \mathbf{h}_{n-j}^{ \pm} \times\left.\mathbf{n}\right|_{0^{ \pm}} & \text {on } \quad \Gamma,
\end{array}
$$

where $\left.\cdot\right|_{ \pm \frac{1}{2}}$ abbreviates the trace on $Y_{3}= \pm \frac{1}{2}$, and $\mathbf{h}_{n}^{ \pm}$denotes the trace of $\mathbf{H}_{n}^{ \pm}$on $\Gamma_{ \pm}$.

5.3. First terms of the asymptotics. In the previous section we have derived the coupled systems for the terms of the asymptotic expansions to any order $n$. Hence we can determine now the first terms $\underline{\mathfrak{T}}_{n}=\left(\mathfrak{H}_{n}, \mathfrak{h}_{n}\right)$ and $\mathbf{H}_{n}$ by induction. 
The coupled system of order 1 . For $n=0$ in the previous system, we find that $\underline{\mathfrak{H}}_{0}=\left(\mathfrak{H}_{0}, \mathfrak{h}_{0}\right)$ and $\mathbf{H}_{0}$ satisfy

$$
\begin{aligned}
\gamma^{2} \mathfrak{h}_{0} & =0 & & \text { in } \Gamma \times I, \\
\operatorname{curl} \mathbf{H}_{0}^{ \pm} & =\mathbf{j}_{ \pm} & & \text {in } \Omega_{ \pm}, \\
\operatorname{div} \mathbf{H}_{0}^{ \pm} & =0 & & \text { in } \Omega_{ \pm}, \\
\left.\mathbf{H}_{0}^{ \pm} \cdot \mathbf{n}\right|_{0^{ \pm}} & =\left.\frac{\mu_{o}}{\mu_{ \pm}} \mathfrak{h}_{0}\right|_{ \pm \frac{1}{2}} & & \text { on } \Gamma, \\
\mathbf{H}_{0}^{+} \cdot \mathbf{n} & =0 & & \text { on } \partial \Omega, \\
-\partial_{3}^{2} \mathfrak{H}_{0, \alpha}+\gamma^{2} \mathfrak{H}_{0, \alpha} & =0 & & \text { in } \Gamma \times I, \\
\left.\mathfrak{H}_{0}\right|_{ \pm \frac{1}{2}} & =\mathbf{n} \times \mathbf{h}_{0}^{ \pm} \times\left.\mathbf{n}\right|_{0^{ \pm}} & & \text {on } \Gamma .
\end{aligned}
$$

Obviously, (5.8a) implies with $\gamma \neq 0$ that $\mathfrak{h}_{0}=0$ and in view of (5.8b), (5.8c), (5.8d) and (5.8e) we can assert that the magnetic field satisfies the PMC boundary conditions, and we obtain the limit system (3.1)-(3.2) for $\mathbf{H}_{0}^{ \pm}$. Then the unique solution of the ODE (5.8f)-(5.8g) is, with the choice (3.5) for $\gamma$, the tangential field

$$
\underline{\mathfrak{Y}}_{0,0}\left(y_{\beta}\right) \cosh \left(\gamma Y_{3}\right)+\underline{\mathfrak{Y}}_{0,1}\left(y_{\beta}\right) \sinh \left(\gamma Y_{3}\right)
$$

with

$$
\underline{\mathfrak{Y}}_{0,0}=\frac{1}{\cosh \left(\frac{\gamma}{2}\right)}\left\{\mathbf{h}_{0}\right\}_{\Gamma} \quad \text { and } \quad \underline{\mathfrak{Y}}_{0,1}=\frac{1}{2 \sinh \left(\frac{\gamma}{2}\right)}\left[\mathbf{h}_{0}\right]_{\Gamma} .
$$

The coupled system of order 2 . Then in the same way as above we find that $\mathfrak{H}_{1}=\left(\mathfrak{H}_{1}, \mathfrak{h}_{1}\right)$ and $\mathbf{H}_{1}$ satisfy

$$
\begin{aligned}
\gamma^{2} \mathfrak{h}_{1} & =-L_{3}^{1}\left(\underline{\mathfrak{Y}}_{0}\right) & & \text { in } \Gamma \times I, \\
\operatorname{curl} \mathbf{H}_{1}^{ \pm} & =0, & & \text { in } \quad \Omega_{ \pm}, \\
\operatorname{div} \mathbf{H}_{1}^{ \pm} & =0 & & \text { in } \Omega_{ \pm}, \\
\left.\mathbf{H}_{1}^{ \pm} \cdot \mathbf{n}\right|_{0^{ \pm}} & =\left.\left.\frac{\mu_{o}}{\mu_{ \pm}} \mathfrak{h}_{1}\right|_{ \pm \frac{1}{2}} \mp \frac{1}{2} \partial_{h} \mathbf{H}_{0}^{ \pm} \cdot \mathbf{n}\right|_{0^{ \pm}} & & \text {on } \Gamma, \\
\mathbf{H}_{1}^{+} \cdot \mathbf{n} & =0 & & \text { on } \quad \partial \Omega, \\
-\partial_{3}^{2} \mathfrak{H}_{1, \alpha}+\gamma^{2} \mathfrak{H}_{1, \alpha} & =-L_{\alpha}^{1}\left(\underline{\mathfrak{Y}}_{0}\right) & & \text { in } \Gamma \times I, \\
\left.\mathfrak{H}_{1}\right|_{ \pm \frac{1}{2}} & =\mathbf{n} \times \mathbf{h}_{1}^{ \pm} \times\left.\mathbf{n}\right|_{0^{ \pm}} \pm \frac{1}{2} \partial_{h} \mathbf{n} \times \mathbf{h}_{0}^{ \pm} \times\left.\mathbf{n}\right|_{0^{ \pm}} & & \text {on } \Gamma .
\end{aligned}
$$

The normal component $\mathfrak{h}_{1}$ of the profile $\underline{\mathfrak{H}}_{1}$ (of order 1 ) is given by equation (5.10a). According to (5.9) and using (5.4) we obtain

$$
\mathfrak{h}_{1}\left(y_{\beta}, Y_{3}\right)=-\gamma^{-1}\left(D_{\alpha}\left\{\mathrm{h}_{0}^{\alpha}\right\}\left(y_{\beta}\right) \frac{\sinh \left(\gamma Y_{3}\right)}{\cosh \left(\frac{\gamma}{2}\right)}+D_{\alpha}\left[\mathrm{h}_{0}^{\alpha}\right]\left(y_{\beta}\right) \frac{\cosh \left(\gamma Y_{3}\right)}{2 \sinh \left(\frac{\gamma}{2}\right)}\right)
$$

where $h_{0}^{\alpha}$ denote the tangential components of $\mathbf{h}_{0}$. 
Now, inserting this explicit representation into the conditions (5.10d), we find that the term $\mathbf{H}_{1}^{-}$solves the following boundary value problem :

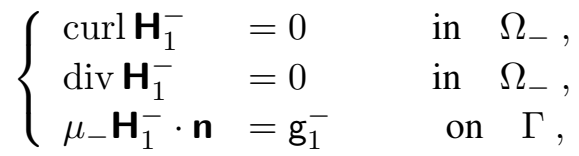

and the term $\mathbf{H}_{1}^{+}$satisfies the problem

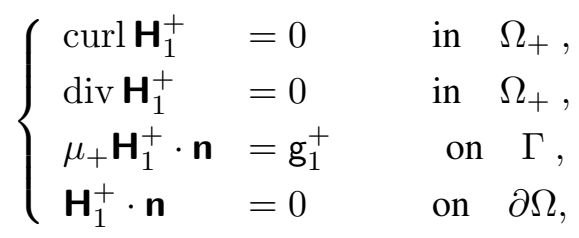

with

$$
\mathrm{g}_{1}^{ \pm}:=\left.\left.\mu_{o} \mathfrak{h}_{1}\right|_{ \pm \frac{1}{2}} \mp \frac{\mu_{ \pm}}{2} \partial_{h} \mathbf{H}_{0}^{ \pm} \cdot \mathbf{n}\right|_{0^{ \pm}}
$$

i.e.

$$
\mathrm{g}_{1}^{ \pm}=-\left.\gamma^{-1} \mu_{o}\left( \pm D_{\alpha}\left\{\mathrm{h}_{0}^{\alpha}\right\}\left(y_{\beta}\right) \tanh \left(\frac{\gamma}{2}\right)+D_{\alpha}\left[\mathrm{h}_{0}^{\alpha}\right]\left(y_{\beta}\right) \frac{1}{2 \tanh \left(\frac{\gamma}{2}\right)}\right) \mp \frac{\mu_{ \pm}}{2} \partial_{h} \mathbf{H}_{0}^{ \pm} \cdot \mathbf{n}\right|_{0^{ \pm}}
$$

The next term which is determined is the tangential field $\mathfrak{H}_{1}$. The unique solution of the ODE $(5.10 \mathrm{f})-(5.10 \mathrm{~g})$ is

$$
\mathfrak{H}_{1}\left(y_{\beta}, Y_{3}\right)=\left(a_{0}\left(y_{\beta}\right)+Y_{3} b_{0}\left(y_{\beta}\right)\right) \cosh \left(\gamma Y_{3}\right)+\left(a_{1}\left(y_{\beta}\right)+Y_{3} b_{1}\left(y_{\beta}\right)\right) \sinh \left(\gamma Y_{3}\right)
$$

with

$$
b_{0, \alpha}=H \mathfrak{H}_{0,0 ; \alpha}-b_{\alpha}^{\beta} \mathfrak{H}_{0,0 ; \beta} \quad \text { and } \quad b_{1, \alpha}=H \mathfrak{H}_{0,1 ; \alpha}-b_{\alpha}^{\beta} \mathfrak{H}_{0,1 ; \beta},
$$

and (recalling that $\left.\mathbf{h}_{\mathbf{T}}=\mathbf{n} \times\left(\mathbf{h}_{\mid \Gamma} \times \mathbf{n}\right)\right)$

$$
a_{0}=-\frac{1}{2} \tanh \left(\frac{\gamma}{2}\right) b_{1}+\frac{1}{\cosh \left(\frac{\gamma}{2}\right)}\left(\left\{\mathbf{h}_{1, \mathbf{T}}\right\}_{\Gamma}+\frac{1}{4}\left[\partial_{h} \mathbf{h}_{0, \mathbf{T}}\right]_{\Gamma}\right),
$$

and

$$
a_{1}=-\frac{1}{2} \operatorname{coth}\left(\frac{\gamma}{2}\right) b_{0}+\frac{1}{2 \sinh \left(\frac{\gamma}{2}\right)}\left(\left[\mathbf{h}_{1, \mathbf{T}}\right]_{\Gamma}+\left\{\partial_{h} \mathbf{h}_{0, \mathbf{T}}\right\}_{\Gamma}\right)
$$

5.4. Further notes for deriving the second order condition. We have explained in Sec. 2.4 guidelines to derive impedance conditions from the boundary conditions (2.4d) on $\Gamma$ for the terms of the asymptotic expansions. It is convenient to write the impedance conditions with mean and jump traces.

One may think that the term $\partial_{h} \mathbf{H}_{0}^{ \pm} \cdot \mathbf{n}$ in (5.13), as it is neither a Dirichlet nor a Neumann trace of $\mathbf{H}_{0}^{ \pm} \in \mathbf{H}\left(\operatorname{curl}, \Omega_{ \pm}\right)$, is not suitable to use in variational formulations or the finite element method. However, using the relation (recalling that $\left.\mathbf{u}_{\mathbf{T}}=\mathbf{n} \times\left(\mathbf{u}_{\mid \Gamma} \times \mathbf{n}\right)\right)$

$$
\operatorname{div} \mathbf{u}=\operatorname{div}_{\Gamma} \mathbf{u}_{\mathbf{T}}+\partial_{h}(\mathbf{u} \cdot \mathbf{n})+\mathbf{u} \cdot \mathbf{n} \operatorname{div} \mathbf{n},
$$

since we have the equalities $\mathbf{H}_{0}^{ \pm} \cdot \mathbf{n}=0$ and $\operatorname{div} \mathbf{H}_{0}^{ \pm}=0$ on $\Gamma$, we can write

$$
\partial_{h} \mathbf{H}_{0}^{ \pm} \cdot \mathbf{n}=-\operatorname{div}_{\Gamma} \mathbf{H}_{0, \mathbf{T}}^{ \pm} \quad \text { on } \Gamma \text {. }
$$


Since we can write the last term $\operatorname{div}_{\Gamma} \mathbf{H}_{0, \mathbf{T}}^{ \pm}$with the covariant derivative

$$
\operatorname{div}_{\Gamma} \mathbf{H}_{\mathbf{T}}=D_{\alpha} \mathrm{h}^{\alpha} \quad \text { on } \Gamma,
$$

we find that the right hand side in (5.13) can be written as

$$
\mathrm{g}_{1}^{ \pm}=-\gamma^{-1} \mu_{o}\left( \pm \operatorname{div}_{\Gamma}\left\{\mathbf{H}_{0, \mathbf{T}}\right\} \tanh \left(\frac{\gamma}{2}\right)+\operatorname{div}_{\Gamma}\left[\mathbf{H}_{0, \mathbf{T}}\right] \frac{1}{2 \tanh \left(\frac{\gamma}{2}\right)}\right) \pm \frac{\mu_{ \pm}}{2} \operatorname{div}_{\Gamma} \mathbf{H}_{0, \mathbf{T}}^{ \pm}
$$

Finally the boundary conditions for $\mathbf{H}_{1}$ on $\Gamma$ in (5.12) can be written as

$$
\left[\mu \mathbf{H}_{1} \cdot \mathbf{n}\right]_{\Gamma}=\left(\{\mu\}_{\Gamma}-2 \frac{\mu_{o}}{\gamma} \tanh \left(\frac{\gamma}{2}\right)\right) \operatorname{div}_{\Gamma}\left\{\mathbf{H}_{0, \mathbf{T}}\right\}_{\Gamma}+\frac{1}{4}[\mu]_{\Gamma} \operatorname{div}_{\Gamma}\left[\mathbf{H}_{0, \mathbf{T}}\right]_{\Gamma},
$$

and

$$
\left\{\mu \mathbf{H}_{1} \cdot \mathbf{n}\right\}_{\Gamma}=\left(\frac{\{\mu\}}{4}-\frac{\mu_{o}}{2 \gamma} \operatorname{coth}\left(\frac{\gamma}{2}\right)\right) \operatorname{div}_{\Gamma}\left[\mathbf{H}_{0, \mathbf{T}}\right]_{\Gamma}+\frac{1}{4}[\mu]_{\Gamma} \operatorname{div}_{\Gamma}\left\{\mathbf{H}_{0, \mathbf{T}}\right\}_{\Gamma}
$$

where we have used the equalities $\{\mathbf{A}\}_{\Gamma}=\{\mu\}_{\Gamma}\left\{\frac{1}{\mu} \mathbf{A}\right\}_{\Gamma}+\frac{1}{4}[\mu]_{\Gamma}\left[\frac{1}{\mu} \mathbf{A}\right]_{\Gamma}$ and $[\mathbf{A}]_{\Gamma}=\{\mu\}_{\Gamma}\left[\frac{1}{\mu} \mathbf{A}\right]_{\Gamma}+$ $[\mu]_{\Gamma}\left\{\frac{1}{\mu} \mathbf{A}\right\}_{\Gamma}$ for any vector field $\mathbf{A}$.

The impedance conditions (3.3d) of order 2 are then obtained by adding the previous equations multiplied by $\varepsilon$ to the PMC conditions $\left\{\mathbf{H}_{0} \cdot \mathbf{n}\right\}_{\Gamma}=\left[\mathbf{H}_{0} \cdot \mathbf{n}\right]_{\Gamma}=0$ (see (3.1c) and (3.2c)) for $\mathbf{H}_{0}$ and by replacing $\mathbf{H}_{0}+\varepsilon \mathbf{H}_{1}$ on the left hand side by the new unknown $\mathbf{H}_{\varepsilon}^{1}$ and by replacing $\varepsilon \mathbf{H}_{0}$ on the right hand side by $\varepsilon \mathbf{H}_{\varepsilon}^{1}$. 


\section{REFERENCES}

[1] A. Alonso Rodríguez, P. Fernandes, and A. Valli. Weak and strong formulations for the time-harmonic eddycurrent problem in general multi-connected domains. European J. Appl. Math., 14(4):387-406, 2003.

[2] C. Amrouche, C. Bernardi, M. Dauge, and V. Girault. Vector potentials in three-dimensional non-smooth domains. Math. Methods Appl. Sci., 21(9):823-864, 1998.

[3] H Bateman. The Mathematical Analysis of Electrical and Optical Wave-Motion on the basis of Maxwell's Equations. Cambridge University Press, 1915.

[4] O. Biro, K. Preis, K.R. Richter, R. Heller, P. Komarek, and W. Maurer. FEM calculation of eddy current losses and forces in thin conducting sheets of test facilities for fusion reactor components. IEEE Trans. Magn., 28(2):1509-1512, 1992.

[5] A. Bossavit. Electromagnétisme, en vue de la modélisation, volume 14 of Mathématiques \& Applications (Berlin) [Mathematics \& Applications]. Springer-Verlag, Paris, 1993.

[6] A. Buffa, H. Ammari, and J.-C. Nédélec. A justification of eddy currents model for the Maxwell equations. SIAM Journal on Applied Mathematics, 60(5):1805-1823, 2000.

[7] G. Caloz, M. Dauge, E. Faou, and V. Péron. On the influence of the geometry on skin effect in electromagnetism. Comput. Methods Appl. Mech. Engrg., 200(9-12):1053-1068, 2011.

[8] M. Costabel. A Remark on the Regularity of Solutions of Maxwell's Equations on Lipschitz Domains. Mathematical Methods in the Applied Sciences, 12(4):365-368, 1990.

[9] M. Costabel, M. Dauge, and S. Nicaise. Singularities of eddy current problems. ESAIM: Mathematical Modelling and Numerical Analysis, 37(5):807-831, 2003.

[10] M. Duruflé, V. Péron, and C. Poignard. Thin layer models for electromagnetism. Commun. Comput. Phys., 16(1):213-238, 2014.

[11] K. O. Friedrichs. Differential forms on riemannian manifolds. Communications on Pure and Applied Mathematics, 8(4):551-590, 1955.

[12] C. Geuzaine, P. Dular, and W. Legros. Dual formulations for the modeling of thin electromagnetic shells using edge elements. IEEE Trans. Magn., 36(4):799-803, 2000.

[13] V. Girault and P.A. Raviart. Finite element methods for Navier-Stokes equations: theory and algorithms. Springer series in computational mathematics. Springer-Verlag, 1986.

[14] R. Hiptmair. Symmetric coupling for eddy current problems. SIAM J. Numer. Anal., 40(1):41-65, 2002.

[15] H. Igarashi, A. Kost, and T. Honma. Impedance boundary condition for vector potentials on thin layers and its application to integral equations. Eur. Phys. J. AP, 1:103-109, 1998.

[16] J.-M. Jin, J. L. Volakis, C.L. Yu, and A.C. Woo. Modeling of resistive sheets in finite element solutions. IEEE Trans. Antennas and Propagation, 40(6):727-731, 1992.

[17] E.F. Knott and T.B.A. Senior. Non-specular radar cross section study. Technical Report 011764-1-T, Univ. Michigan. Radiation Lab., Ann Arbor, MI, USA, Jan 1974.

[18] L. Krähenbühl and D. Muller. Thin layers in electrical engineering. example of shell models in analysing eddycurrents by boundary and finite element methods. IEEE Trans.Mag., 29(2):1450-1455, 1993.

[19] T. Levi-Civita. La teoria elettrodinamica di Hertz di fronte ai fenomeni di induzione. Rend. Lincei (5), 11(2):7581, 1902. (in Italian).

[20] R. C. MacCamy and E. Stephan. A skin effect approximation for eddy current problems. Arch. Rational Mech. Anal., 90(1):87-98, 1985.

[21] R.C. MacCamy and E. Stephan. Solution procedures for three-dimensional eddy current problems. Journal of mathematical analysis and applications, 101(2):348-379, 1984.

[22] I.D. Mayergoyz and G. Bedrosian. On calculation of 3-D eddy currents in conducting and magnetic shells. IEEE Transactions on Magnetics, 31(3):1319-1324, 1995.

[23] William McLean. Strongly Elliptic Systems and Boundary Integral Equations. Cambridge University Press, 2000.

[24] J. McWhirter. Computation of three-dimensional eddy currents in thin conductors. IEEE Trans. Magn., 18(2):456-460, 1982.

[25] T. Nakata, N. Takahashi, K. Fujiwara, and Y. Shiraki. 3D magnetic field analysis using special elements. IEEE Trans. Magn., 26(5):2379-2381, 1990.

[26] J-C. Nédélec. Acoustic and electromagnetic equations : integral representations for harmonic problems. Applied mathematical sciences. Springer, New York, 2001. 
[27] V. Péron, K. Schmidt, and M. Duruflé. Equivalent transmission conditions for the time-harmonic Maxwell equations in 3D for a medium with a highly conductive thin sheet. SIAM Journal on Applied Mathematics, 76(3):1031-1052, 2016.

[28] J. Poltz and K. Romanowski. Solution of quasi-stationary field problems by means of magnetic scalar potential. IEEE Trans. Magn., 19(6):2425-2428, 1983.

[29] D. Rodger and N. Atkinson. Finite element method for 3D eddy current flow in thin conducting sheets. IEE Proceedings A, 135(6):369-374, 1988.

[30] K. Schmidt and A. Chernov. A unified analysis of transmission conditions for thin conducting sheets in the time-harmonic eddy current model. SIAM J. Appl. Math., 73(6):1980-2003, 2013.

[31] K. Schmidt and A. Chernov. Robust transmission conditions of high order for thin conducting sheets in two dimensions. IEEE Trans. Magn., 50(2):41-44, 2014.

[32] K. Schmidt and R. Hiptmair. Asymptotic boundary element methods for thin conducting sheets. Discrete Contin. Dyn. Syst. Ser. S, 8(3):619-647, 2015.

[33] T. Senior. Approximate boundary conditions. IEEE Trans. Antennas and Propagation, 29(5):826-829, 1981.

[34] O.V. Tozoni and I.D. Mayergoyz. Analysis of three-dimensional electromagnetic fields. Technika, Kiev, 1974. in russian.

LMAP CNRS UMR 5142 \& TEAM MAGIQUE 3D INRIA BordeAuX SUd-OUEST, UniVERsité DE PAU et des Pays de L’Adour, AVEnue de L'Université, BP 1155, 64013 Pau CEdex, France 